\title{
Infrared Spatial Interferometer
}

Charles Townes, Manfred Bester, William Danchi, David Hale, John Monnier, et al.

Charles H. Townes, Manfred Bester, William C. Danchi, David D. Snyder Hale, John D. Monnier, Everett A. Lipman, Peter G. Tuthill, Mark A. Johnson, Donald L. Walters, "Infrared Spatial Interferometer," Proc. SPIE 3350, Astronomical Interferometry, (24 July 1998); doi: 10.1117/12.317159

Event: Astronomical Telescopes and Instrumentation, 1998, Kona, HI, United States 


\title{
Infrared Spatial Interferometer
}

\author{
C.H. Townes ${ }^{a}$, M. Bester ${ }^{a}$, W.C. Danchi ${ }^{a}$, D.D.S. Hale ${ }^{a}$, J.D. Monnier ${ }^{a}$, E.A. Lipman ${ }^{a}$, \\ P.G. Tuthill ${ }^{\mathrm{a}}$, M.A. Johnson ${ }^{\mathrm{b}}$, and D. Walters ${ }^{\mathrm{c}}$

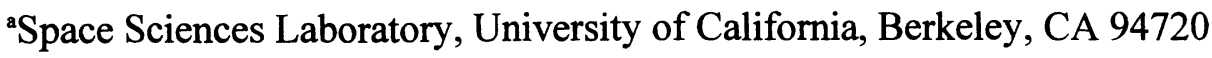 \\ ${ }^{b}$ Lawrence Livermore National Laboratory, Livermore, CA

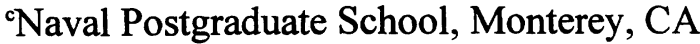

\begin{abstract}
The Infrared Spatial Interferometer (ISI) is an interferometer installed on Mt. Wilson and operating in the $10 \mu \mathrm{m}$ wavelength region, using heterodyne detection and two movable $1.65 \mathrm{~m}$ telescopes. Its general technology and characteristics, recent changes, and observational results are broadly discussed. Some compensation for atmospheric path length fluctuations is demonstrated. Stellar observations show, among other characteristics, that many stars emit gas and dust episodically with times of $10-100$ years between events, and that stellar diameters measured in the mid-infrared region are about $10 \%$ larger than those measured with interferometry using visible light.
\end{abstract}

Keywords: stellar interferometry, infrared interferometry

\section{INTRODUCTION}

Since the Berkeley Infrared Spatial Interferometer (ISI) appears to be the only astronomical interferometer operating in the mid-infrared at present, we shall not only describe the instrument and some of its results but discuss the field somewhat broadly. Most interferometry has been done in the radio and visible regions, although there is increasing attention to the near infrared for interferometric work on stars. The mid-infrared allows one to look at rather different objects than do these shorter wavelengths. It detects not only stars and hot dust, but also the mildly warm dust surrounding stars. Mid-infrared wavelengths can also penetrate clouds and see stars which, due to intervening dust, may not be intense enough at shorter wavelengths to study. The longer wavelengths change seeing problems; atmospheric fluctuations of importance vary more slowly and also correlate over much larger distances than those for visible light. The mid-infrared is quite sensitive to changes in temperature of dust clouds which are relatively cool and is useful in detecting such changes, but not very sensitive to changes in the temperatures of stars themselves. For example, a star at $3000 \mathrm{~K}$ will change its visible luminosity by a factor of about 2 if the temperature is changed only $250 \mathrm{~K}$, while wavelengths in the $10 \mu \mathrm{m}$ region change intensity by only about $10 \%$. This means that stars which have a nonuniform surface temperature or a changing one can give a very nonuniform intensity or a changing one in the visible region, while being relatively constant in the infrared. Interferometry at visible wavelengths and in the mid-IR are thus mutually complementary.

\section{DESCRIPTION AND CHARACTERISTICS OF THE ISI EQUIPMENT}

The ISI has been operating on Mt. Wilson, using two telescopes of effective aperture $1.65 \mathrm{~m}$. It has been previously described, ${ }^{1}$ but will be summarized briefly here. The telescopes are in trailers, and hence can be moved to vary their baseline, at present between $4 \mathrm{~m}$ and $35 \mathrm{~m}$. An extension to about $75 \mathrm{~m}$ is planned soon. The two telescopes can be seen in Fig. 1 with a separation of $16.1 \mathrm{~m}$. The design is sometimes called a "Pfund" telescope. It involves a flat 80 " mirror in an alt-azimuth mount and a 65" parabola fixed in position. The flat mirror can be regarded as a coelostat, sending radiation into the parabola which then focuses the radiation through a hole in the flat mirror and onto an optics table. These telescopes are illustrated schematically by Fig. 2. The dashed lines in this figure represent $\mathrm{HeNe}$ laser beams which are distance interferometers. They measure path lengths within the telescope. Once the trailer is positioned, it can be lowered, allowing the two larger mirrors and their mounts, as well as the optics table, to sit on kinematic mounts which in turn are supported by concrete blocks laid into the ground. Thus, the two mirrors and 
optics table are freed from touching the trailer, and movements of operators inside the trailer do not shake them substantially.

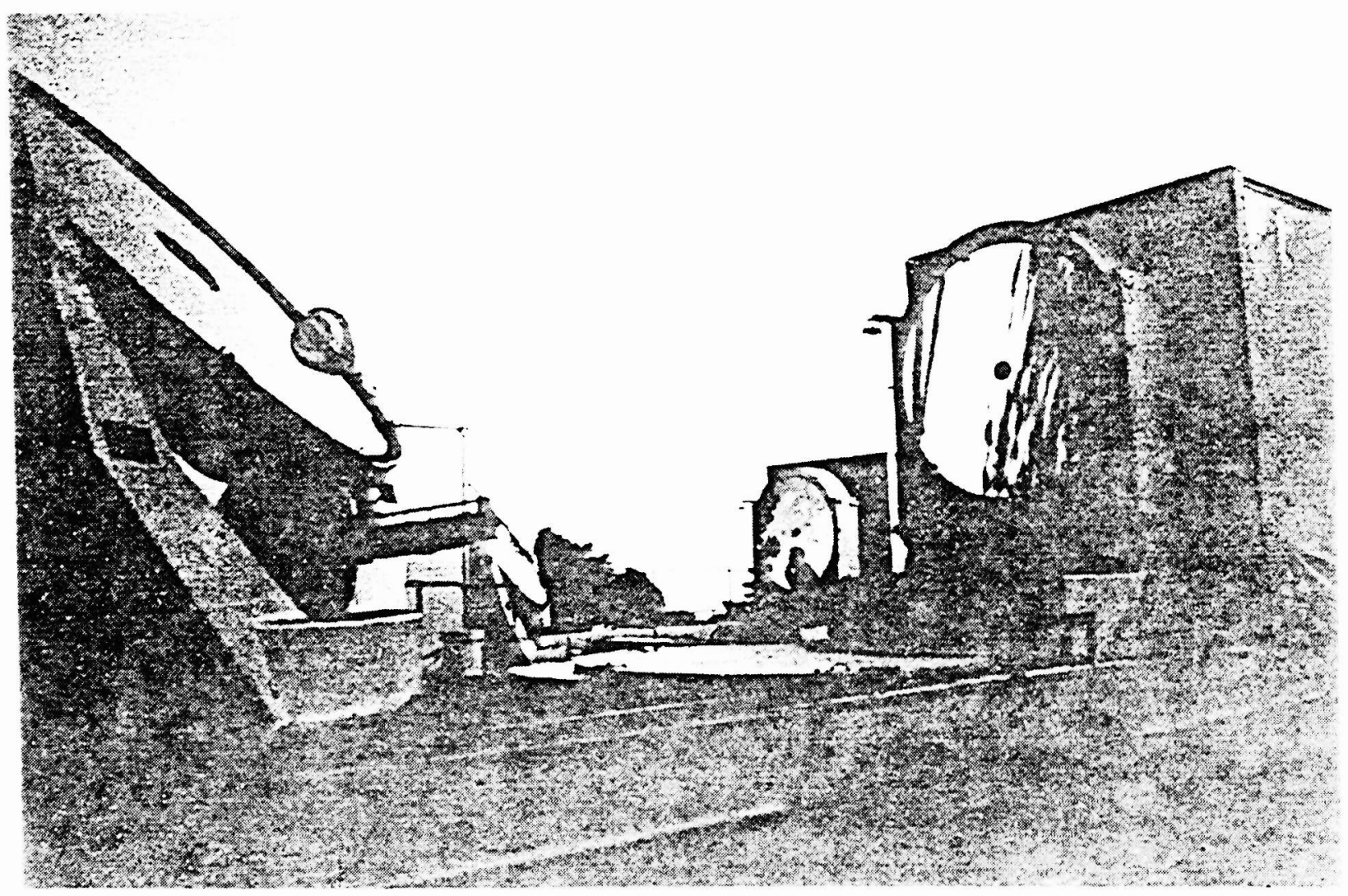

Fig. 1. The two $1.65 \mathrm{~m}$ Pfund telescopes of the ISI, deployed on Mt. Wilson.

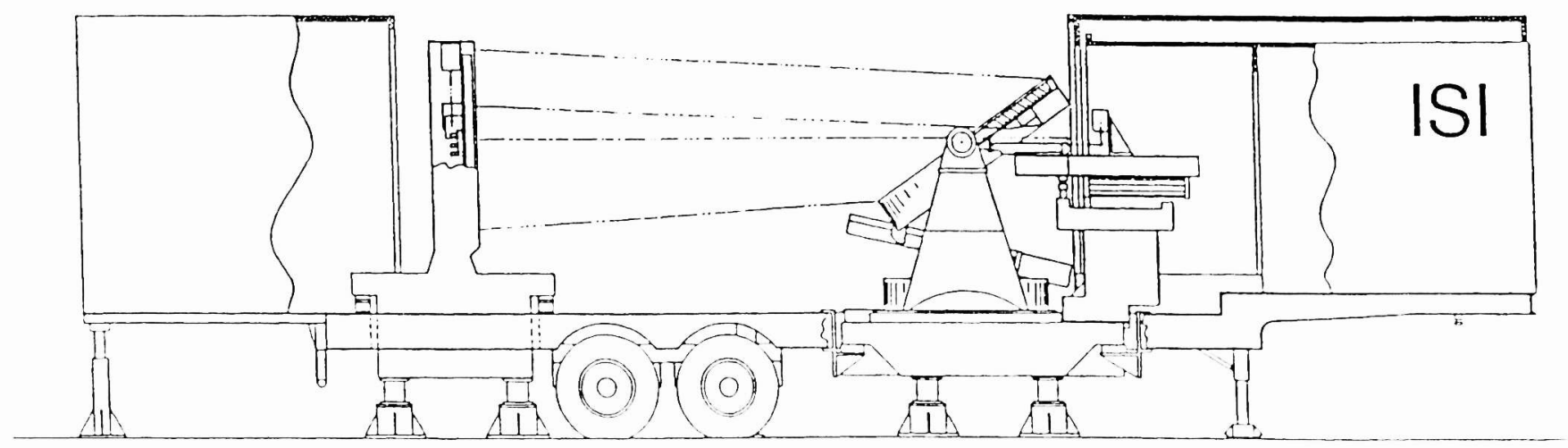

UCB INFRARED SPATIAL INTERFEROMETER

Fig. 2. A schematic of an ISI telescope, with a 65" stationary parabolic mirror and an 80 " flat mirror on an alt-azimuth mount. The optics and instrument table is behind the flat mirror. Dashed lines indicate the beams of the HeNe laser distance interferometers. 
A schematic of the interferometer operation is shown in Fig. 3. Heterodyne detection is used with a $\mathrm{CO}_{2}$ laser as a local oscillator. The local oscillator power is mixed with the incoming infrared astronomical signal in a detector which provides a beat frequency between the two with an IF bandwidth of about $3 \mathrm{GHz}$. Both sidebands, each 3 $\mathrm{GHz}$ wide, are then transmitted by $\mathrm{RF}$ cable and mixed with a similar signal from the other telescope, providing a beat between the two signals or an interference fringe. Each telescope has its own local oscillator, and a beam sent from the $\mathrm{CO}_{2}$ laser in one telescope to the second telescope allows the second $\mathrm{CO}_{2}$ laser to be locked in phase to the first. However, the phase lock is done with a frequency difference of about $1 \mathrm{MHz}$ frequency, which is varied in such a way as to give what is known as lobe rotation. This provides a fringe signal of fixed frequency as the observed object moves across the sky. This type of heterodyne detection and lobe rotation is commonly used in radio astronomy. In fact the whole system is in principle essentially like a radio interferometer.

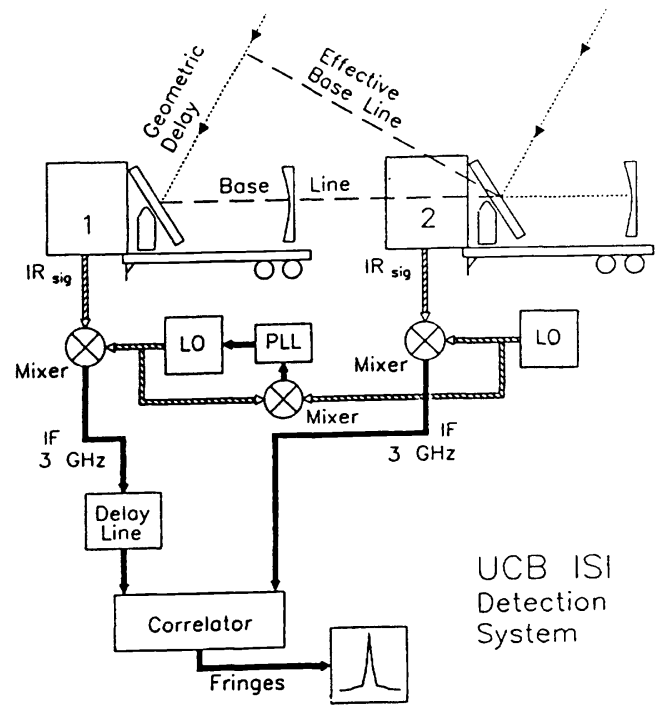

Fig. 3. A schematic of the ISI interferometer, showing the local oscillators (LO) and the semiconductor detector-mixers which provide heterodyne detection. The two LOs are locked together in phase by the phase lock loop (PLL) which responds to their beat signal in another detector-mixer. The IF signals from the two telescopes, of bandwidth $3 \mathrm{GHz}$, are beat together to form a "fringe."

The laser beam sent from the first telescope to the second one is reflected back along the same path and beat with the outgoing signal in the first telescope. This allows automatic pathlength adjustment to produce a path length which is an exact multiple of the wavelength, thus assuring that the phase of the local oscillator in the second telescope is uniquely related to that in the first.

The interference fringe produced by mixing the signals from the two telescopes is initially at a frequency of about $1 \mathrm{MHz}$, but it is mixed with a signal which differs from it by about $100 \mathrm{~Hz}$, and the final fringe is observed at their beat frequency near $100 \mathrm{~Hz}$. This frequency varies slightly with time depending on fluctuations in the relative atmospheric pathlengths.

A power spectrum of an interference fringe for the star $\alpha$ Orionis is shown in Fig. 4. This represents a signal recorded over a time of 512 seconds. The width of the central spike is about $1 / 500 \mathrm{~Hz}$, the width produced by the finite time of observation. Small spikes on either side of the central fringe represent additional frequencies of the fringe spectrum produced by atmospheric fluctuations. This particular fringe was taken during excellent seeing with the two telescopes separated by $4 \mathrm{~m}$, and provided remarkably small phase fluctuations due to a very constant atmosphere. Fig. 5 shows a contrasting example, with a very wide fringe produced by large and rapid atmospheric fluctuations. As can be seen, the width of this fringe is about $15 \mathrm{~Hz}$, indicating that the phase of the fringe changes substantially on a timescale of about $1 / 15$ of a second. This is very poor seeing. The timescale for changes of an optical fringe is shorter than that for wavelengths of $11 \mu \mathrm{m}$, which is our normal working wavelength, by a factor somewhat greater than the ratio of the two wavelengths. Thus, this particular fringe was taken when an optical fringe would have been changing on a timescale somewhat shorter than $1 / 300$ of a second. Nevertheless, the total 
power of the fringe and hence the visibility of $\alpha$ Orionis can be determined from this very wide fringe associated with very poor seeing simply by integrating the total power under the curve, which can be done with substantial accuracy in spite of its breadth. Thus, while the signal to noise ratio of the fringe in Fig. 4 is obviously very high, the integrated signal to noise ratio with the very poor seeing shown in Fig. 5 is still fairly high.

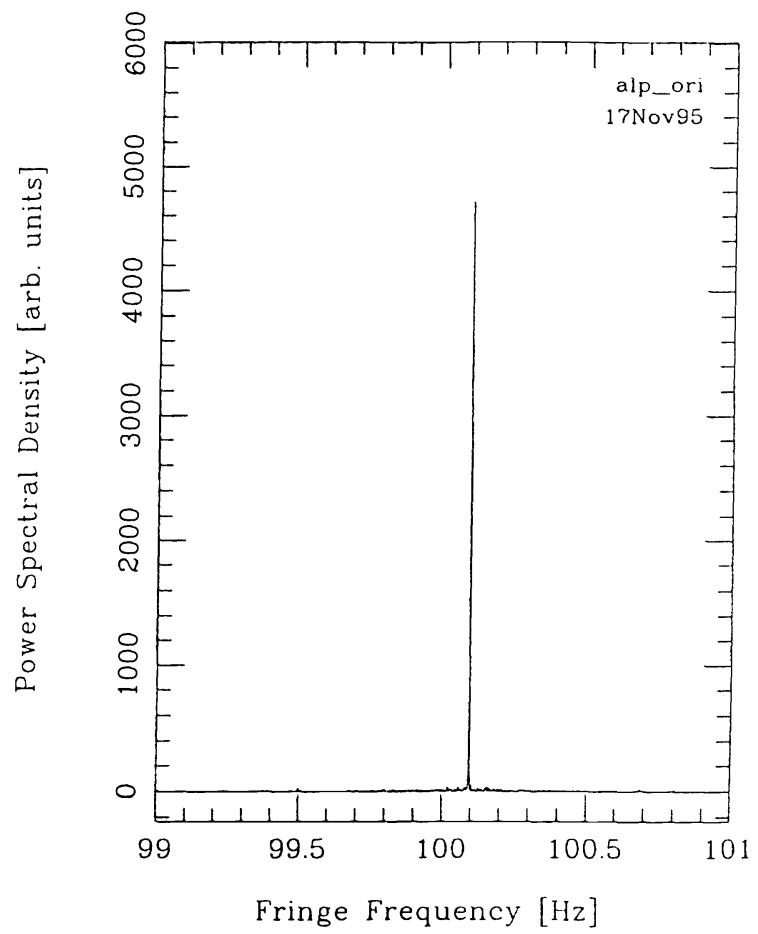

Fig. 4. An interference fringe signal of $\alpha$ Orionis under exceptionally good seeing conditions. Atmospheric fluctuations are small enough that only very weak sidebands over a small fraction of one Hertz are produced by their variations. The data were taken for 512 seconds, and the very narrow resulting fringe has a width due primarily to this finite time of measurement.

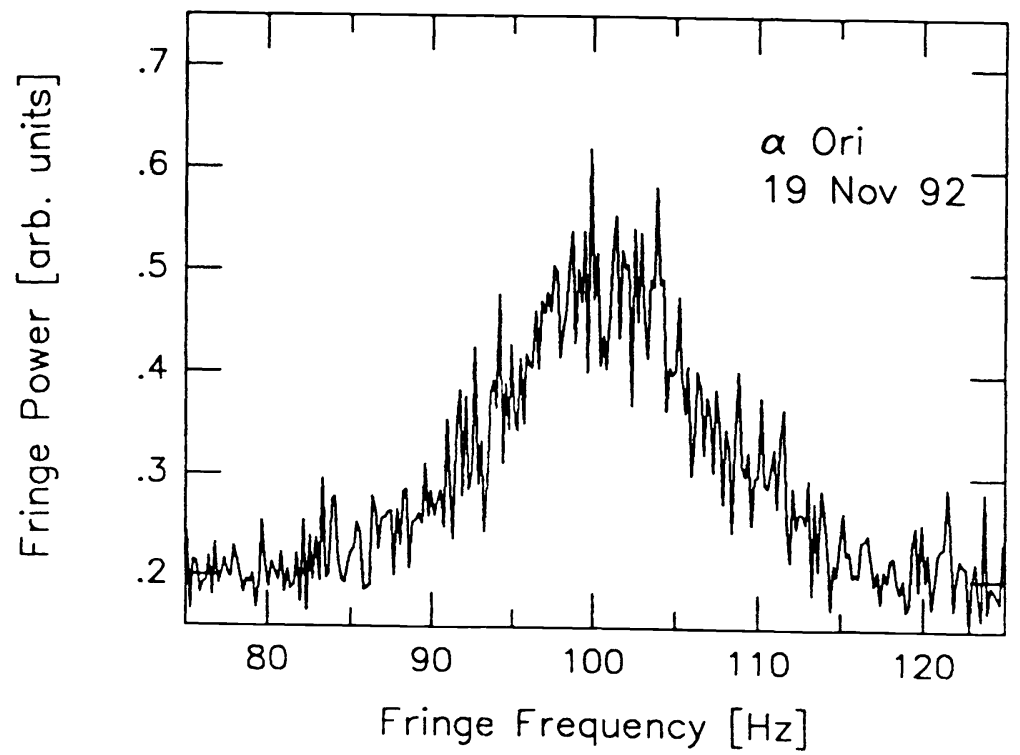

Fig. 5. An interference fringe from $\alpha$ Orionis under very poor seeing conditions. In this case the atmospheric pathlength is fluctuating severely and on timescales comparable with the inverse width seen, or about $1 / 15 \mathrm{sec}$. This is fast for wavelengths as integrating the total powe of such fluctuations, the fringe magnitude, or total power, can be rather accurately determined by 


\section{SENSITIVITY CONSIDERATIONS}

It is perhaps useful to make some comparison at this point between heterodyne detection and direct detection for the measurement of fringes or interferometric visibilities. In the radio region, essentially all detection is done by heterodyne methods, since no direct photon detection is normally practical. At optical wavelengths, direct photon detection is normal and generally advisable for interferometry. Heterodyne detection produces a detector noise of 1 photon per second per unit bandwidth for one polarization. For optical photons, this would be indeed a great deal of noise. In the radio region, there is background radiation of many photons per second per unit bandwidth in any case, and heterodyne detection provides no substantial additional noise. The mid-infrared is a region where both types of detection are appropriate, depending on the exact purpose and usage of such detection.

It can be argued that direct detection is much more sensitive than heterodyne detection in the mid-infrared, and hence that it is most appropriately used for interferometry. The number of photons per second per spatial mode per unit bandwidth due to thermal radiation at room temperature is about 0.03 . If transmission through the atmosphere and the optical system used is reasonably good, then not more than perhaps $60 \%$ or 0.02 photons per second are received as background noise. This would imply that for a given bandwidth, the sensitivity is greater by $\sqrt{1 / 0.02}$, or about 7 , for direct detection than for heterodyne. In addition, heterodyne detection covers a rather narrow frequency range, in our particular case about $0.2 \mathrm{~cm}^{-1}$, and it is unlikely to be larger by more than a few factors of 2 . For direct detection one can easily use a bandwidth 100 times greater, implying another factor of 10 advantage in sensitivity for direct detection. However, in actual practice various phenomena or interferometer uses can substantially modify the apparent advantage of direct detection.

If a number of telescopes are used in an interferometric system in order to produce images, the energy must be divided up if direct detection is used for a number of baselines. Thus, for 10 telescopes, the energy from each telescope must be divided into 9 equal parts, which then makes a relative loss in the sensitivity of a factor of 9 . For heterodyne detection, the division produces no such loss, because amplification and division of the signal can be done with no decrease in signal to noise ratio. In addition, optical delay lines are rather more complex and troublesome than are the delay lines for the narrow bandwidths used in heterodyne detection. In our case, the delay lines are simply RF cables. Instead of tracking the atmospheric fluctuations, we need only to put in calculated changes in the delay line length associated with the geometry of the telescopes and the object observed. To vary the delay, we switch in or out $1 \mathrm{~cm}$ lengths of delay line, thus providing an accuracy of $1 / 2 \mathrm{~cm}$ for the delay, which is adequate. Thus the arrangement of delay lines, particularly for use of many telescopes, is rather simpler and more convenient for the heterodyne case, and avoids losses in signal to noise ratio due to division.

If detection is attempted on a rather weak star with no stronger star near enough to be used for tracking the necessary delay, then the apparent advantage of direct detection decreases substantially further. This is because the appropriate pathlength must be tracked on a timescale comparable with the time of change of phase due to atmospheric fluctuations, which can be as short as about $1 / 100$ second. Thus, an adequate signal must be obtained in $1 / 100$ of a second, unless some other special tracking mechanism is used, such as search and averaging when the fringe cannot be locked on. In the case of heterodyne detection, the delay is not varied by tracking on the star, but by precalculation, so that the fringe signal can be accumulated for periods as long as an hour or more, possibly averaging over more than one night. This gives the possibility of averaging a weak signal for a much longer time. If, for example, some allowance is made for averaging a fringe signal even when it is not phase-tracked perfectly, one might assume a time as long as 1 second for detecting a weak fringe with a direct detection system. Comparing this with an hour for a heterodyne system gives heterodyne detection a relative sensitivity gain of a factor of 60 . However, this conclusion applies only in some cases. If there is a bright star sufficiently close to the source observed, then this problem for direct detection can be eliminated.

A further helpful characteristic of heterodyne detection is that the detected radiation, from which the total power is determined, is only that component of the astronomical signal which is in a single spatial mode and thus overlaps the laser radiation correctly. This is the same part of the power which can produce interference between signals from two telescopes, so that seeing has little effect on the visibility calibration. For direct detection, radiation in only a single mode is sometimes similarly obtained by passing the radiation through a single mode fiber, and a 
similar advantage in visibility measurement is achieved. However, this use of a fiber entails a loss, usually by a factor between 2 and 5, which is another factor reducing the relative sensitivity advantage of direct detection from its theoretical ideal.

For carrying out interferometry on spectral lines, it is necessary to use bandwidths somewhat narrower than the line widths. Doppler widths at $10 \mu \mathrm{m}$ wavelength for material around stars are typically about $0.02 \mathrm{~cm}^{-1}$. For bandwidths this small or smaller, non-fundamental noise in direct detectors such as leakage currents or leakage radiation limits their sensitivity. Heterodyne detection by contrast does not become less sensitive than its fundamental limits as the bandwidth is narrowed. Thus for bandwidths as narrow as $0.02 \mathrm{~cm}^{-1}$ or less, heterodyne detection can be more sensitive than direct detection even without allowing for the various special factors noted above. For interferometry on spectral lines, this characteristic is an important one.

The above discussion illustrates why probably both direct and heterodyne detection systems should be developed for mid-infrared interferometry. They will be competitive and may serve complementary functions.

\section{ATMOSPHERIC PATH LENGTH FLUCTUATIONS}

Atmospheric pathlength fluctuations, closely related to "seeing," represent an important problem for interferometry from the Earth's surface. For that reason we have paid some attention to their measurement, sources, and the possibility of correction. Such studies have shown that there is a correlation between fluctuations measured within the telescope optics by the HeNe laser distance interferometers (LDI) and fluctuations along the pathlengths through the atmosphere above the telescopes. Furthermore, a substantial fraction of the atmospheric pathlength fluctuations is concentrated near the surface. ${ }^{12}$ At Mt. Wilson, under normal good seeing conditions about $60 \%$ of the seeing problem is concentrated within about $30 \mathrm{~m}$ of the surface, and much of it is correlated with fluctuations which are measurable a modest distance above the ground. The ground layer of turbulence is well demonstrated by measurements with a pulsing acoustic radar. Fig. 6 shows the results of such measurements. The pulse length is about $2.5 \mathrm{~m}$, and pulses scattered by atmospheric turbulence are received at ground level. Fig. 6 represents the relative intensity of this received acoustic signal as a function of height in meters and of time in seconds. Pulses are released periodically with 1 second separation. The left-hand part of Fig. 6 shows the turbulence structure of the atmosphere at a particular time on Mt. Wilson in the middle of the day. It can be seen that strong turbulence extends up to at least $140 \mathrm{~m}$ in this case. The right hand part shows the same type of measurement during relatively good seeing in the evening. Here, the scattered radiation is fairly intense up to a distance of about $40 \mathrm{~m}$ and then decreases, demonstrating the ground layer of turbulence.
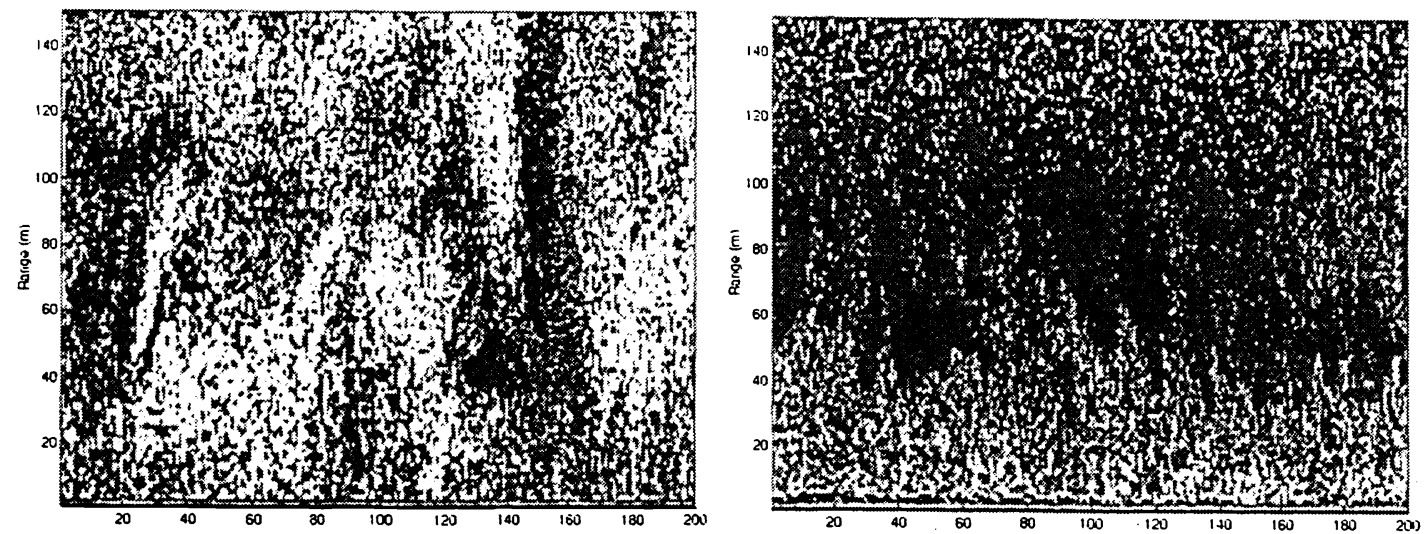

Fig. 6. Scattering of acoustic pulses from the ground by atmospheric turbulence at various heights. Pulses are repeated every second, and shown for a total time of 200 seconds (the horizontal axis). The strength of scattering as a function of height (in meters) above the ground (the vertical axis) is indicated by brightness in this diagram. The left-hand figure represents results during midday on Mt. Wilson, when seeing is poor. The right-hand figure represents results during relatively good seeing conditions during the evening. It demonstrates that atmospheric turbulence is most severe near the ground and up to a height of about $40 \mathrm{~m}$. 
HeNe LDI Phase in Telescope 1, 2 and 1-2
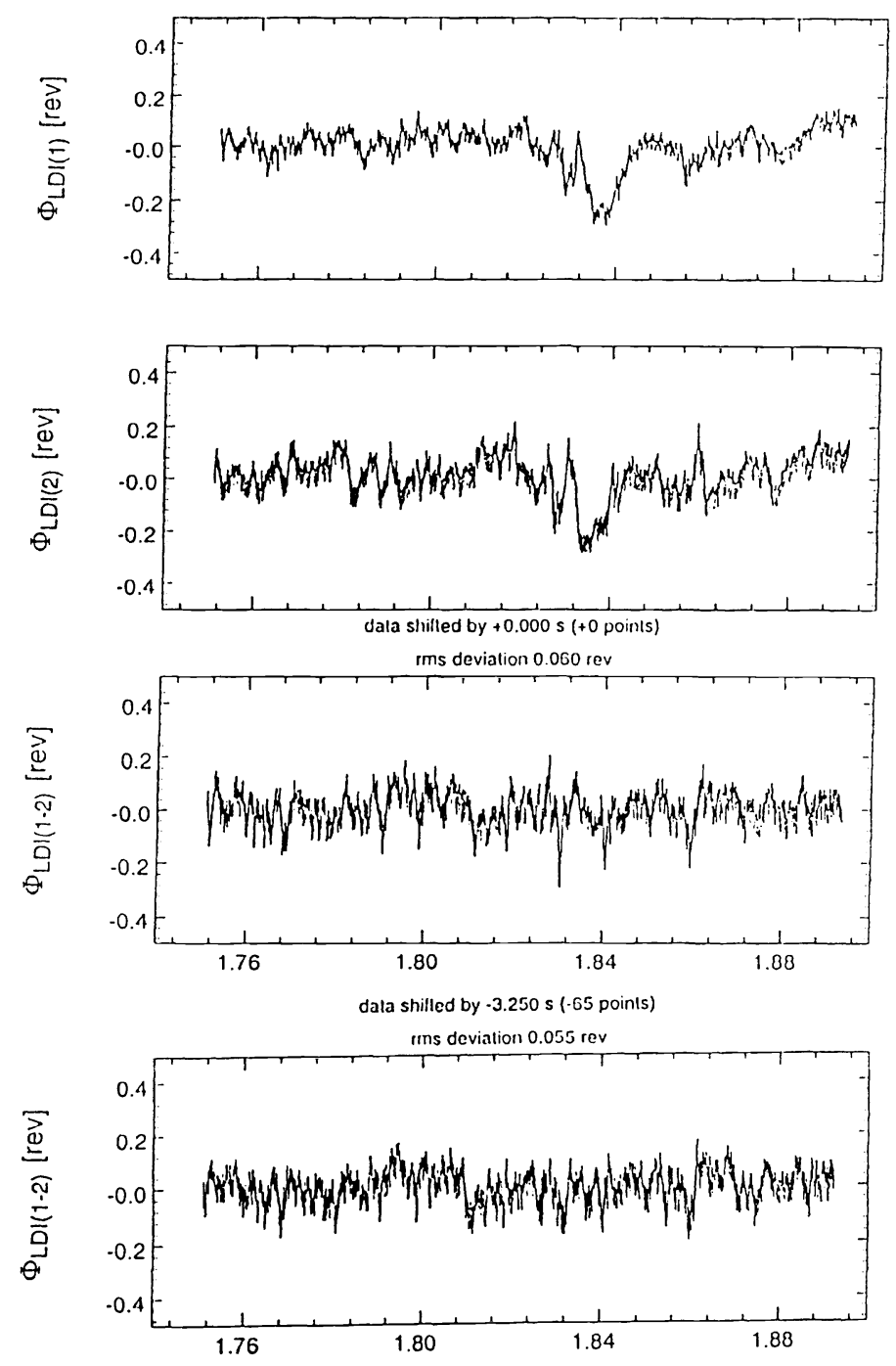

UTC [h]

Fig. 7. Path length fluctuations measured by HeNe laser interferometers within the optical paths between the two large mirrors of each telescope. The top trace shows, for the first telescope, fluctuations in units of $11 \mu \mathrm{m}$ as a function of time (the horizontal axis) over a period of 0.15 hours, or about 10 mins. The second from top trace shows the same measurements for the second telescope during the same period of time. The third from top trace represents the difference between fluctuations in the two telescopes, showing a strong correlation between the two as can be seen visually from the upper two traces. The lowest trace represents the difference between fluctuations in the two telescopes with fluctuations in the first telescope shifted by 3.25 seconds in time with respect to those in the second telescope. This indicates a still stronger correlation if time required for a given volume of air to move from the first to the second telescope is allowed for. 
It is important to note that many of the larger path length variations are correlated over some distance both laterally on the ground and vertically into the atmosphere. Fig. 7 shows variations within the HeNe LDI interferometers in two different telescopes spaced $4 \mathrm{~m}$ apart. The two top traces represent fluctuations measured in wavelengths in the two separate telescopes. The third diagram from the top shows the difference between these two. It can be seen that many of the larger fluctuations are closely similar for the two. The bottom trace represents the difference between paths in the two telescopes when one of them is shifted in time by 3.25 seconds. This shows a still closer correlation between the two, indicating a time delay between fluctuations in the two telescopes. Since the distance between the two is $4 \mathrm{~m}$, this time delay corresponds to a wind of about $2.5 \mathrm{mph}$.

The correlation between fluctuations measured by the two HeNe LDIs within the optics of the telescope and the pathlength difference from the star to the two telescopes is shown in Fig. 8. Here, it can be seen that for the larger and slower fluctuations, the two sets are closely correlated. Those associated with the fringe, or relative pathlengths from the star to the two telescopes, are about 2.5 times larger than what is measured in the $10 \mathrm{~m}$ distance within the telescopes themselves. This directly indicates a correlation up to a distance of about $25 \mathrm{~m}$.

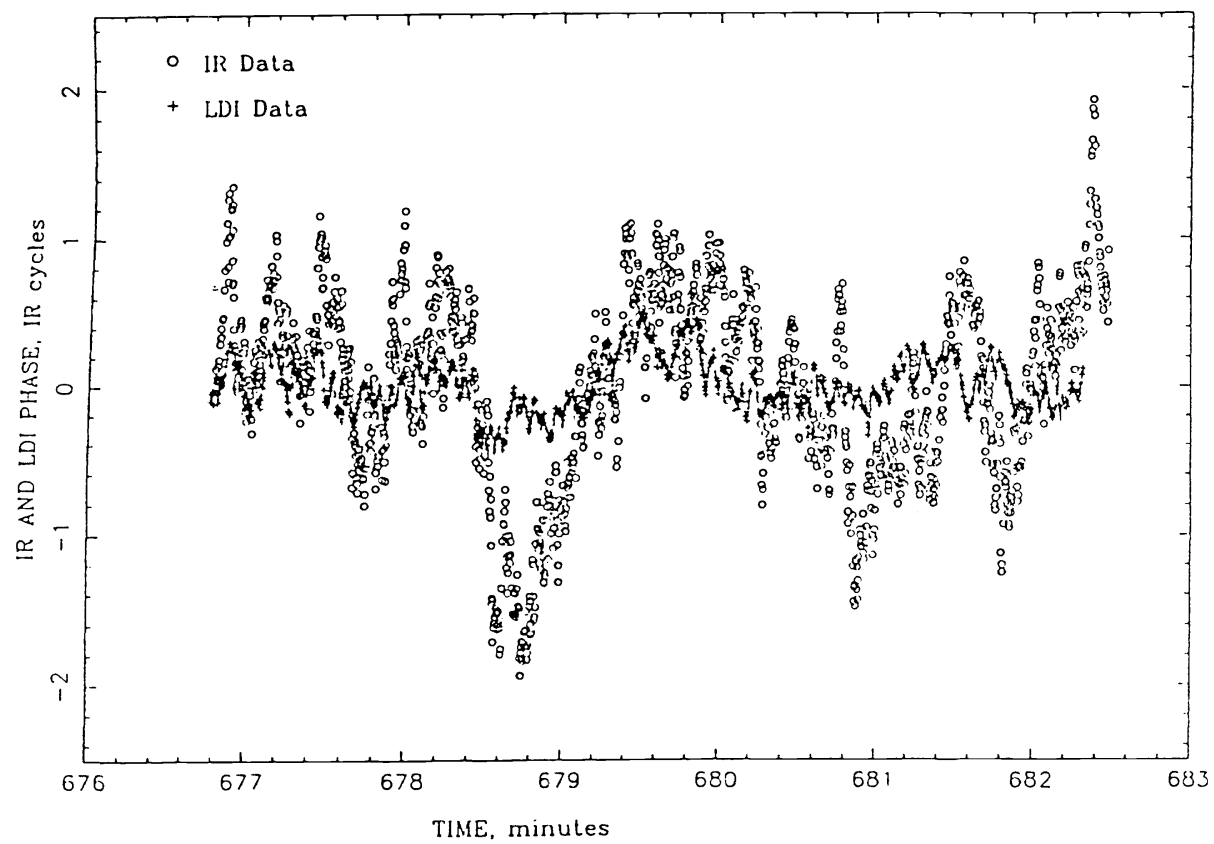

Fig. 8. A comparison between optical path length difference fluctuations within the two telescopes (a distance of $10 \mathrm{~m}$ ) and measured by the HeNe laser distance interferometers (LDI), and those from a star to the detectors. A strong correlation for the larger and slower variations can be seen, with path length fluctuations for the star being about 2.5 times larger in size. This indicates a correlation of fluctuations within the telescope optics near the ground and in the atmosphere above the telescopes.

A relatively simple attempt to correct fringe phase fluctuations due to atmospheric pathlength changes is shown in Fig. 9. Here, the first figure shows a fringe and its many sidebands extending to about $\pm 1 / 2 \mathrm{~Hz}$. The central plot in this figure shows the same fringe when the measured fluctuations within the two telescopes have been subtracted from the fringe's phase fluctuations. It is obviously somewhat stronger at the central frequency and narrower in frequency spread. The third illustration in Fig. 9 shows the fringe fluctuations if twice the fluctuations within the telescopes have been subtracted. It can be seen there that many of the slower fluctuations corresponding to nearby sidebands have been canceled out, and that the central fringe peak has been relatively enhanced. This represents both an increase in signal to noise and an improvement in the possibility of accurate phase measurements. Overall, we believe that substantial refinement and corrections of atmospheric seeing fluctuations can in the long run be achieved, and that this is particularly applicable to the longer infrared wavelengths where the important fluctuations are both slower and of larger scale than those for visible light. 

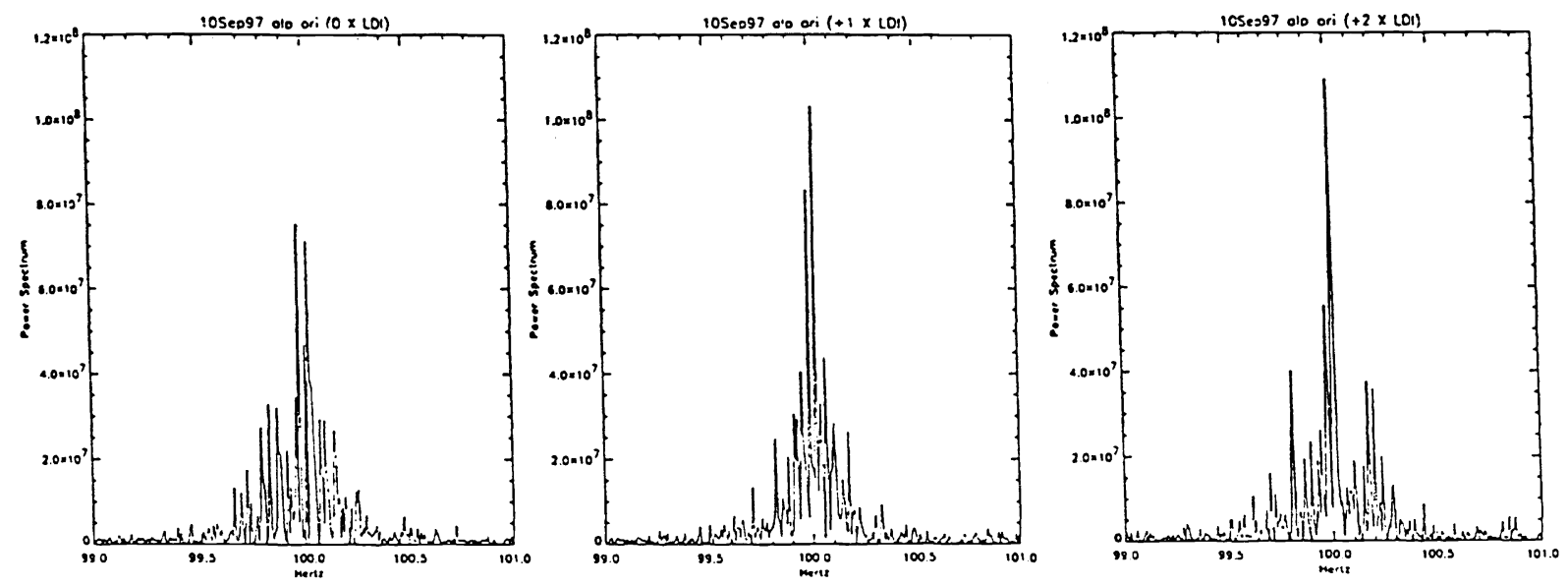

Fig. 9. Correction of fringe phase fluctuations by measurement of atmospheric fluctuations near the ground. The first (left-hand) figure represents the fringe frequency power spectrum after interference of the two $11 \mu \mathrm{m}$ beams from a star to the two telescope detectors. The central figure is the spectrum after subtraction of fluctuations within the telescopes as measured by HeNe laser distance interferometers (LDIs). The third (right-hand) figure represents the fringe spectrum after subtraction of twice the amount of fluctuation measured by the LDIs. This demonstrates the correlation between fluctuations within the telescopes and those in the atmosphere above the telescopes, and that effective correction techniques may be applied to maintain optimum quality of fringes and their phase variations.

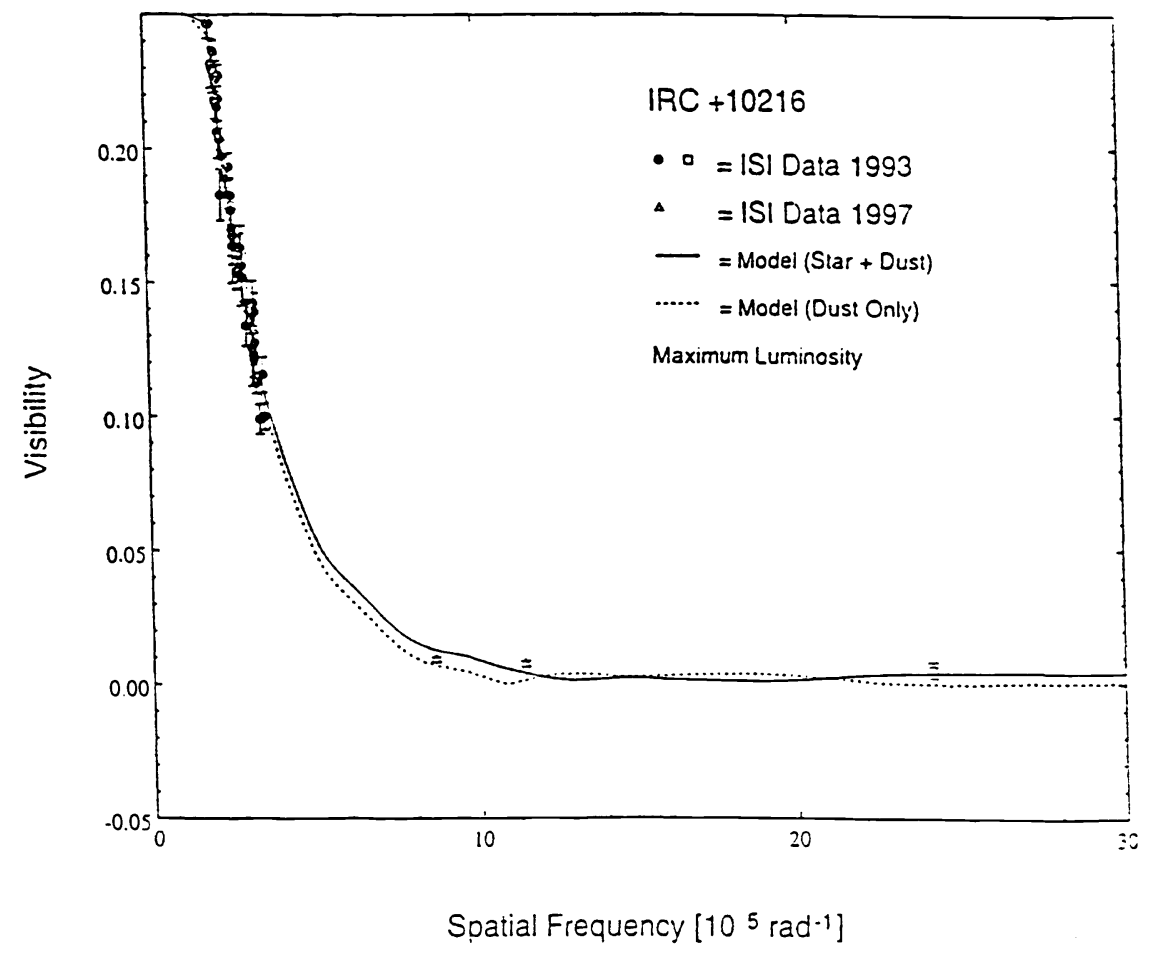

Fig. 10. The $11 \mu \mathrm{m}$ visibility curve of the star IRC +10216 . Radiation from dust around the star is largely resolved when the spatial frequency of fringes is as high as $10^{6} \mathrm{rad}^{-1}$ (1/5 arc second), leaving only a visibility value due to the star itself, which is approximately 0.005 . Thus the star produces only $1 / 200$ of the total $11 \mu \mathrm{m}$ radiation detected. At the lowest resolutions shown, about $75 \%$ of the $11 \mu \mathrm{m}$ radiation is already resolved (visibility $\sim 0.25$ ). This spatial frequency, about $2 \times 10^{5} \mathrm{rad}^{-1}$, corresponds to a resolution of about 0.6 arcseconds. 


\section{STELLAR OBSERVATIONS}

The visibility curve for the bright IR star IRC +10216 is shown in Fig. $10 .^{3}$ Visibility represents simply the ratio of the Fourier spatial transform of intensity across the sky due to the object being observed compared with what it would be for a point source. Thus, at any given spatial frequency and for a simple, non-repetitive pattern, the visibility represents approximately the fraction of the object's radiation which has not been resolved. In this case, a large part of the $11 \mu \mathrm{m}$ radiation has been resolved at our lowest resolutions shown, representing a spatial frequency of about $2 \times 10^{5} \mathrm{rad}^{-1}$, or a resolution of about $1 / 2 \mathrm{arcsec}$. At higher resolutions, additional radiation is resolved until, at a spatial frequency of about $10^{6} \mathrm{rad}^{-1}$, all the dust around the star has been resolved. At the highest resolution shown, corresponding to about 50 milliarcsec (mas), we can expect that only the stellar radiation itself remains unresolved. Its visibility is only 0.005 , representing a very small fraction of the total radiation. Using averaging times of the order of an hour or so, this small visibility can be measured to a precision of about $10 \%$. Radiation at $11 \mu \mathrm{m}$ from the star itself is somewhat larger than this very low visibility would indicate; a model of the dust shell shows that the optical depth of the dust at $11 \mu \mathrm{m}$ is between 1 and 2 . At visible wavelengths, the star has probably never been seen, although a small amount of visible light has been detected which is probably scattered through a somewhat nonuniform dust shell.

Fig. 11 shows a somewhat more detailed picture of the visibility of IRC +10216 . The two curves represent two different phases in the luminosity of the star, which varies on a timescale of about 2 years. The visibility is higher during minimum luminosity because the dust is sufficiently warm to emit $11 \mu \mathrm{m}$ radiation only out to a smaller radius, so that the total radiation is less resolved. However, still another effect is necessary in order to explain this change with phase of the stellar luminosity. This is that new dust must be formed sometime during the cooler period of the star. ${ }^{3}$ The visibility curves indicate a formation of dust in the temperature range 1000 to $1500 \mathrm{~K}$, which is approximately what is expected theoretically for carbonaceous or silicate materials.

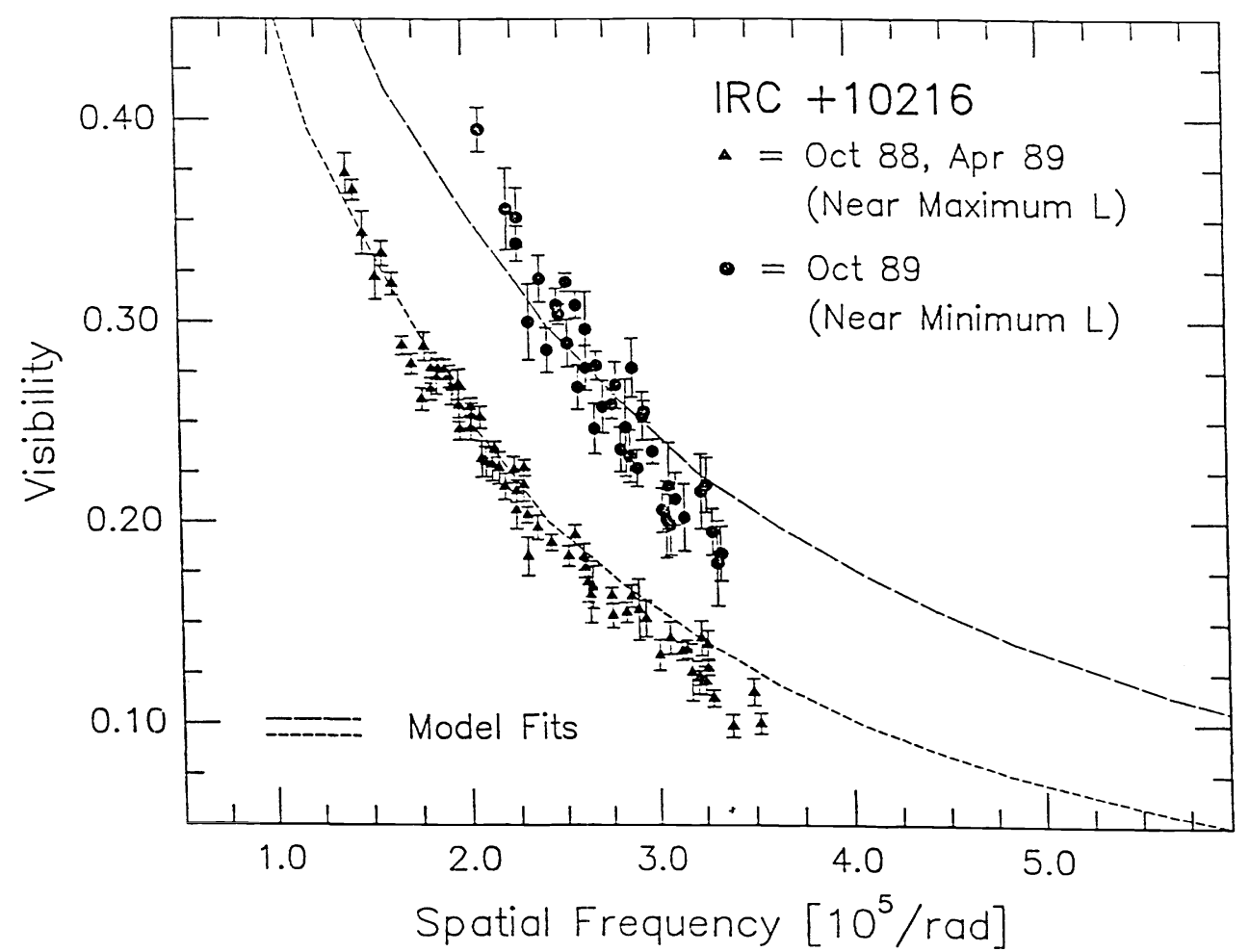

Fig. 11. A detailed plot of the visibility of IRC +10216 showing the visibility curve near maximum and minimum luminosity during the star's approximately 2-year luminosity cycle. Difference between the two curves is due in part to a change in the dust temperature and in part to formation of new dust during the less luminous part of the cycle. 
Another and perhaps simpler case of dust distribution is that around VY CMa, as shown in Fig. 12. The luminosity of VY CMa varies somewhat irregularly and not as much as that of IRC +10216 . It can be seen in Fig. 12 that two measurements, made several years apart, are rather close together. However, some deviation and difference between the two is clear. In this case also, the star itself provides a very small fraction of the total $11 \mu \mathrm{m}$ radiation.

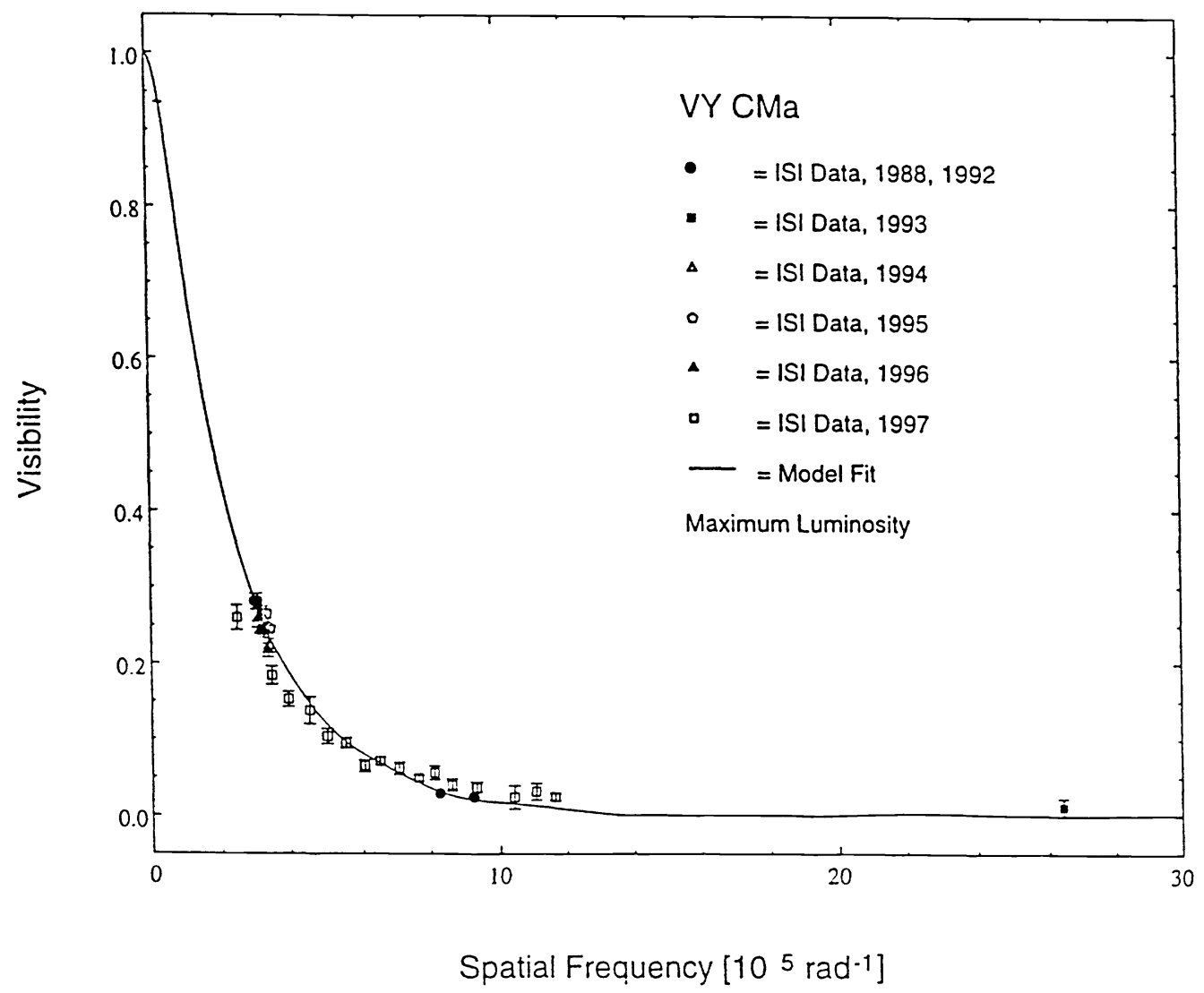

Fig. 12. The $11 \mu \mathrm{m}$ visibility curve for VY CMa, an irregular variable star. The points taken several years apart in time and at various phases of luminosity do not differ markedly, in part because the stellar luminosity does not vary greatly. The star itself, probably largely unresolved at the higher spatial frequencies, contributes only a small fraction of the total $11 \mu \mathrm{m}$ radiation.

It is important to note the complex nature of the dust distribution around such stars and its change with time, which require many measurements over a short period of time in order to describe them fully. If dust flows uniformly from the star, which is frequently assumed, then the dust density should vary as $1 / R^{2}$, where $R$ is the distance from the star. However, the curve fitting in Fig. 11 represents a dust density varying approximately as $1 / \mathrm{R}^{3 / 2}$, which indicates a nonuniform flow. In addition, the dust can vary strongly from spherical symmetry. This is shown clearly by masking-type interferometry in the near IR, which has been carried out on the Keck telescope, as described by Peter Tuthill in a talk at this meeting. The left-hand part of Fig. 13 gives a diagram of the mask used. The right-hand part of Fig. 13 represents the uv plane with the many baselines provided by the mask. Fig. 14 maps the intensity distribution of VY CMa at $2.2 \mu \mathrm{m}$ and $3.1 \mu \mathrm{m}$ as determined by this interferometry. The distribution can be seen to differ strongly from spherical symmetry. The visibility curve of Figs. 12 represents the visibility at 11 $\mu \mathrm{m}$ through such a cloud in one dimension only. The dust shell is probably somewhat more symmetric at $11 \mu \mathrm{m}$ than at $2.2 \mu \mathrm{m}$ and $3.1 \mu \mathrm{m}$ since more distant dust is involved and the variations probably average out to some extent. It is clear, however, that at $11 \mu \mathrm{m}$ it is also important to obtain two dimensional pictures in order to thoroughly study the dust distribution. 

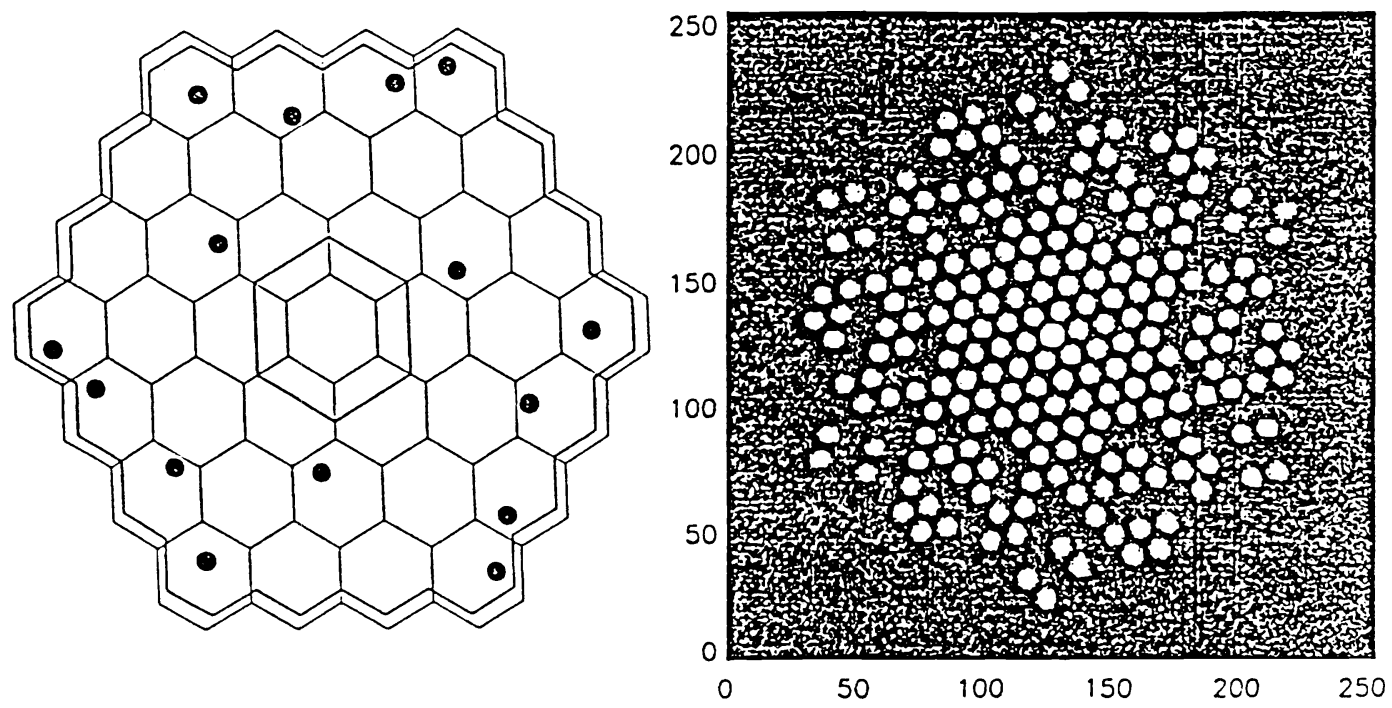

Fig. 13. A mask used for interferometry on the Keck telescope at 2.2 and $3.1 \mu \mathrm{m}$ wavelengths. The left figure shows the position of holes in the mask projected onto the Keck primary mirror. The right figure provides a plot in the UV plane of the many resulting baselines for which interferometry can be obtained.
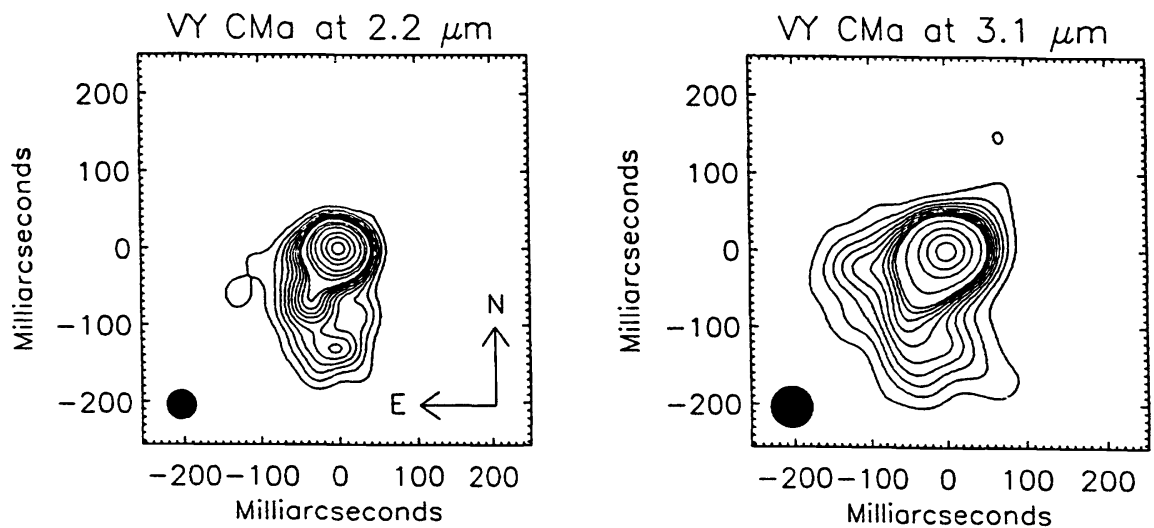

Fig. 14. The intensity distribution of near-IR radiation from VY CMa obtained from masking interferometry on the Keck telescope. The star itself is perhaps in the center of the patterns shown by intensity contours of 2.2 and $3.1 \mu \mathrm{m}$ radiation, not at any one of the intensity peaks. Clearly for this near-IR radiation the intensity distribution deviates markedly from a spherical distribution.

An example in sharp contrast to IRC +10216 and VY Cma is that of $\alpha$ Orionis, for which the visibility curve is shown in Fig. 15. At our lowest resolution of about $1 \mathrm{arcsec}, 40 \%$ of the $11 \mu \mathrm{m}$ radiation is already resolved. For the higher resolutions in this curve, the visibility changes relatively little. This indicates that a dust shell external to the star has been resolved at the lowest resolution, and that there is no further dust between that and the star itself, which is relatively unresolved at the resolutions shown in this figure. The bump near a spatial resolution of $2 \times 10^{5}$ radians $^{-1}$ is believed to be due to the addition in phase of intensity from the star itself and the bright inner edge of the innermost dust shell. This then predicts a radius of about 1 arcsec for the dust shell, a size also indicated by other measurements. ${ }^{3}$ 


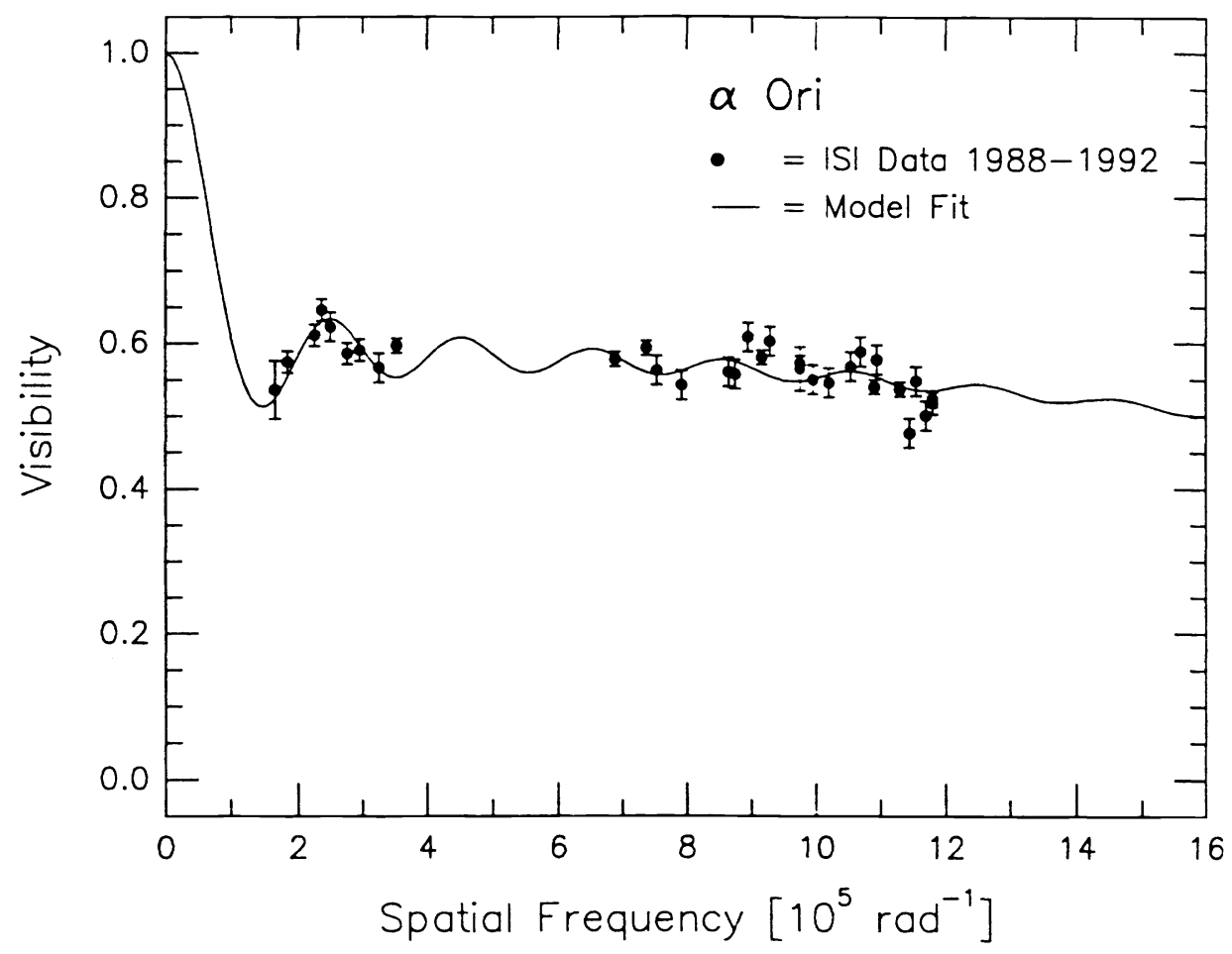

Fig. 15. Visibility curve for $\alpha$ Orionis at $11 \mu \mathrm{m}$. The surrounding dust is rather completely resolved at the lowest resolutions of this curve, and contributes about $40 \%$ of the total $11 \mu \mathrm{m}$ radiation. The slowly decreasing visibility at higher spatial frequencies represents some resolution of the star itself. The bump near a spatial frequency of $2 \times 10^{5} \mathrm{rad}^{-1}$ represents addition of the stellar radiation with that of the inner part of a dust shell, which produces spatial peaks in the visibility curve. This gives a distance from star to shell of about one arcsec.

We have recently measured several stars which are so faint in visible and near infrared radiation that we have not previously been able to track them well. We can now do so thanks to a new near infrared camera which has increased sensitivity, and also to tip-tilt tracking, which allows very fast and relatively accurate tracking. These two new improvements to our system are discussed in the paper given at this meeting by Everett Lipman. One set of data taken with the new camera is shown in Fig. 16, which is the visibility curve for CIT3. In this case, we used three different baselines in a period of about 6 weeks, so that we have a snap shot of the dust distribution of CIT3 at a particular phase of the star. It can be seen that the indicated uncertainties in each point are somewhat larger than what might be guessed from their scatter. This is because part of the uncertainty represents systematic errors rather than random errors from point to point. Thus, the shape of the curve is somewhat more accurate than its absolute value. This visibility curve shows that even at our lowest resolution for this star, about $40 \%$ of the $11 \mu \mathrm{m}$ radiation has been resolved. As resolution is increased, the curve is flat and hence there is no additional dust over some distance until a spatial frequency of about $4 \times 10^{5} \mathrm{rad}^{-1}$, where additional dust begins to be resolved. This indicates an additional dust shell with a diameter of about 250 mas. At much higher resolutions, the curve flattens out indicating that all has been resolved excepting the star itself, and that the star provides a visibility less than about 0.1 . Fig. 17 shows a similar visibility curve taken for IRC +10420 . Here again, about half the total infrared radiation has been resolved at our lowest resolution. There is no additional radiation for some short distance, and then dust begins to be resolved at a spatial frequency of about $2.5 \times 10^{5} \mathrm{rad}^{-1}$, indicating that new dust begins again at a diameter of about $\mathbf{4 0 0}$ mas. In this case the star itself again provides a visibility of less than 0.1 . 


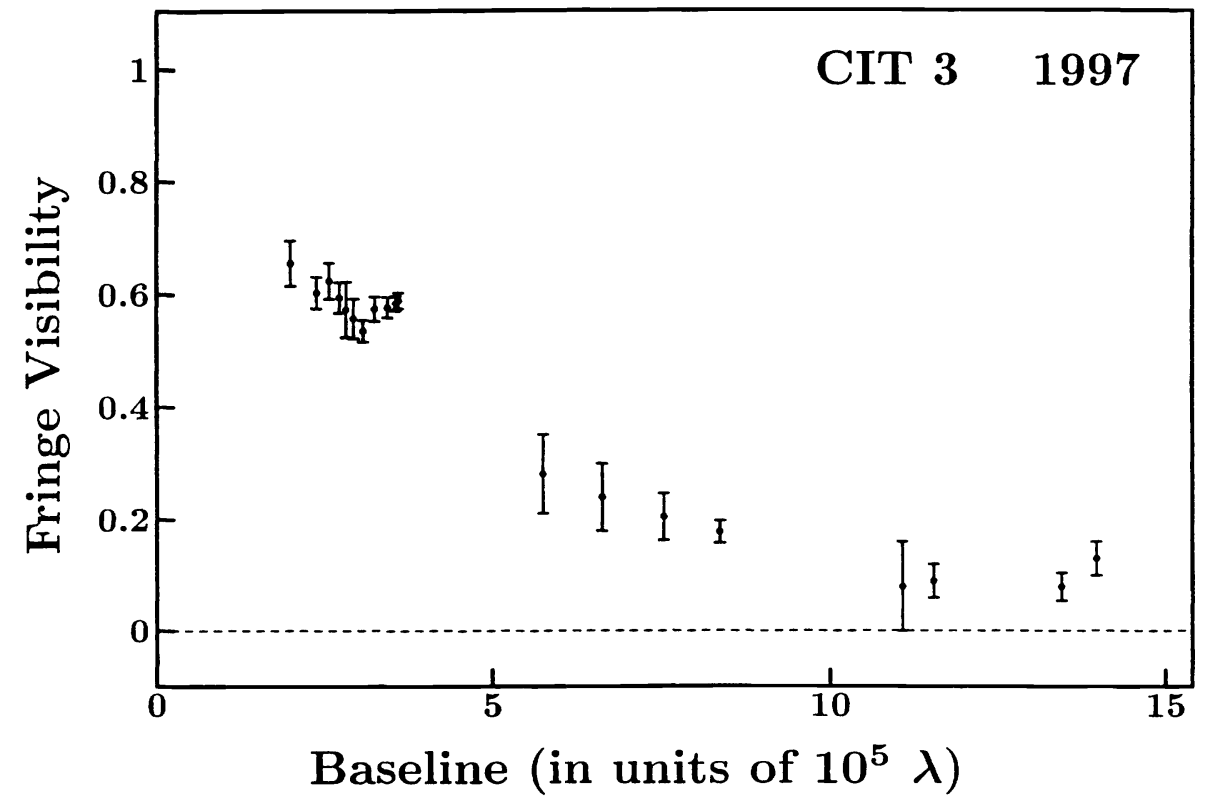

Fig. 16. The $11 \mu \mathrm{m}$ visibility curve of CIT3, showing resolution of one dust shell at the lowest resolutions of the curve, no additional dust resolution before a spatial frequency of about $4 \times 10^{5} \mathrm{rad}^{-1}$, and then resolution of another shell of dust. The inner dust shell has a diameter of about 250 mas.

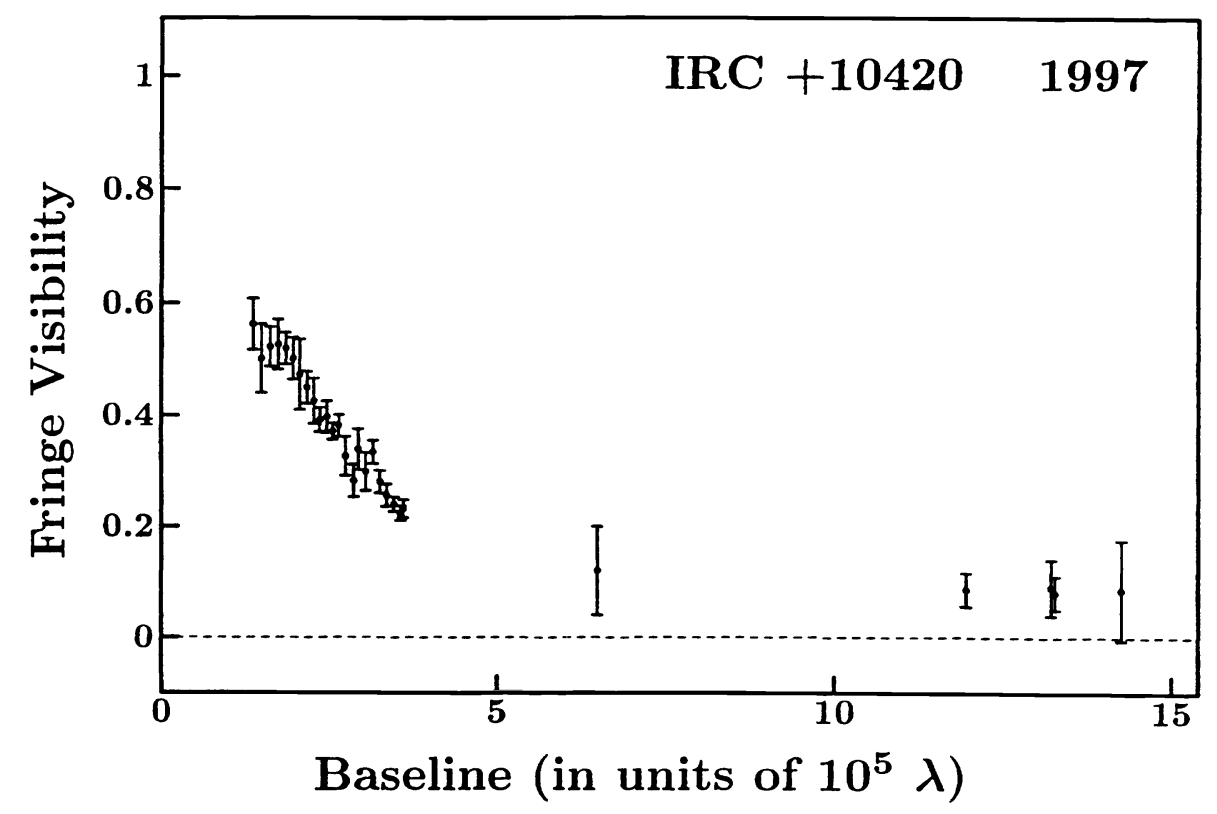

Fig. 17. The $11 \mu \mathrm{m}$ visibility of IRC +10420 , also showing a large outer dust shell, no dust for some distance towards the star, and then resolution of an additional dust shell which has a diameter of about 400 mas. The star itself provides a visibility of about 0.1 , i.e. produces about $10 \%$ of the $11 \mu \mathrm{m}$ radiation. 
Theoretical models of the dust which will fit the preliminary data of Figs. 16 and 17 have yet to be worked out. However, it is immediately clear what qualitative distribution of dust is present. Still another star which these new improvements have allowed us to measure is R Cancri. It turned out to have a visibility of unity for all of our resolutions up to the highest used during last Fall's observations. However, it is clear that there must be dust around this star because of its infrared spectrum. Thus, one can conclude that $\mathrm{R}$ Cancri is surrounded by dust which all lies within a diameter less than about 90 mas. We will in time use higher resolution and measure its distribution more precisely.

Figure 18 shows additional measurements of the $11 \mu \mathrm{m}$ visibility of $\alpha$ Ori. Two new results are obtained from this figure. First, we have used high enough resolution to resolve the star, which is why the curve steadily slopes downward towards the higher resolutions. The theoretical fit for these points allows determination of the diameter of $\alpha$ Orionis, which will be discussed somewhat later. The data also show that visibility at low resolution is substantially different in 1994 from what it was in the period 1988-1992. ${ }^{2}$ The visibility at low resolution in 1994 is somewhat higher and a bit smoother than the earlier curve. This indicates that new dust has been emitted, and is giving additional $11 \mu \mathrm{m}$ radiation fairly near the star. It is resolved at resolutions in the range $15-20 \times 10^{5} \mathrm{rad}^{-1}$. The presence of this new outburst of dust from $\alpha$ Orionis is also indicated by Fig. 19. Here, the visible magnitude and

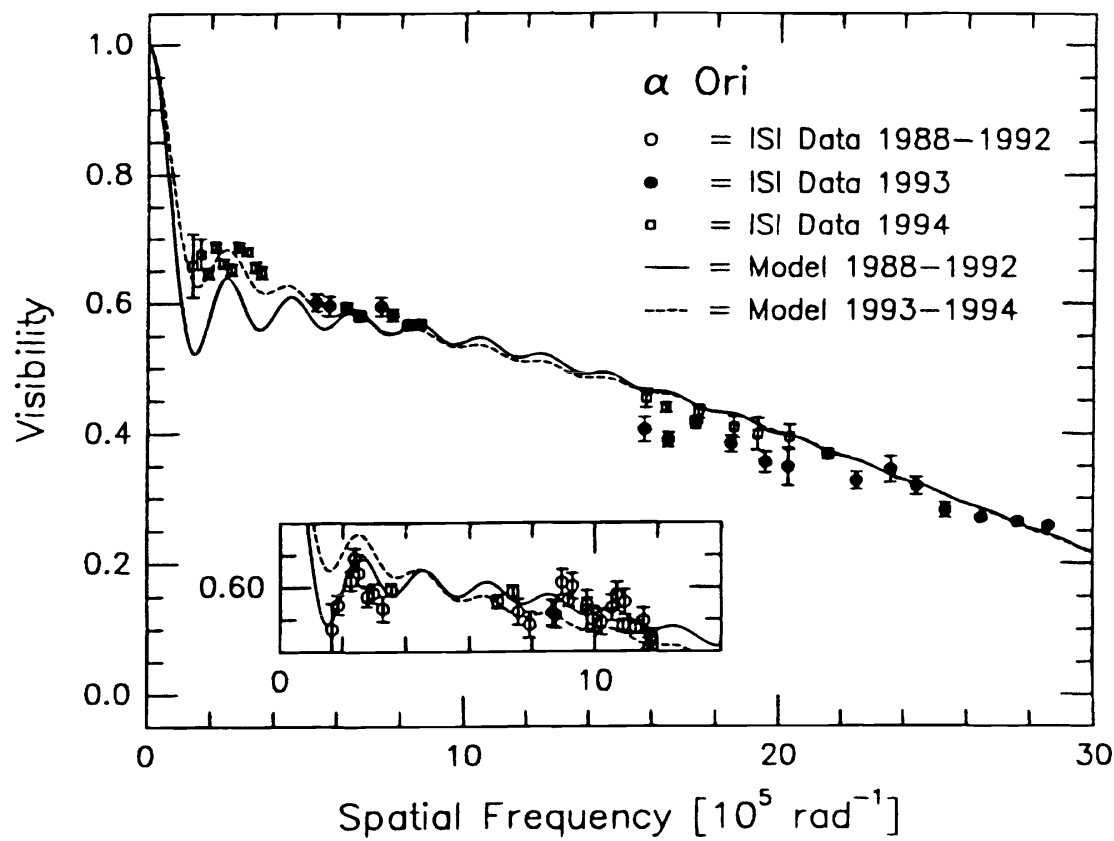

Fig. 18. The $11 \mu \mathrm{m}$ visibility curve of $\alpha$ Orionis. This shows resolution of the star at higher spatial frequencies, which allows determination of its size. In addition, at the lower spatial frequencies the curves taken before and after 1994 differ substantially, indicating emission of a modest amount of additional dust in 1994. The solid and dashed curves represent theoretical fits for a uniform disk of radiation from the star, an outer shell of dust at a radius of about 1 arcsec, and the dashed curve has the addition of some dust near the star, which was emitted in 1994.

$11 \mu \mathrm{m}$ magnitude are compared over the same time period. It is notable that the visible magnitude varies by as much as about $\pm 3 / 10$ of a magnitude. This is partly because a relatively small change in temperature can vary the visible light substantially. The $11 \mu \mathrm{m}$ radiation during most of this period goes up and down a small amount synchronously with the visible radiation. It is much less sensitive to such a temperature change, and this behavior indicates the visible intensity changes are primarily due to temperature changes rather than variation in the stellar diameter. In 1994 and 1995 one can see that the visible magnitude increases sharply while the infrared magnitude by contrast decreases slightly. This indicates that dust has been formed which is screening out some of the visible radiation. We now know that such outbursts or episodic emission of dust are rather typical, which is indicated in part by the distant shell of dust around $\alpha$ Orionis. Such phenomena are also found in many other stars. 


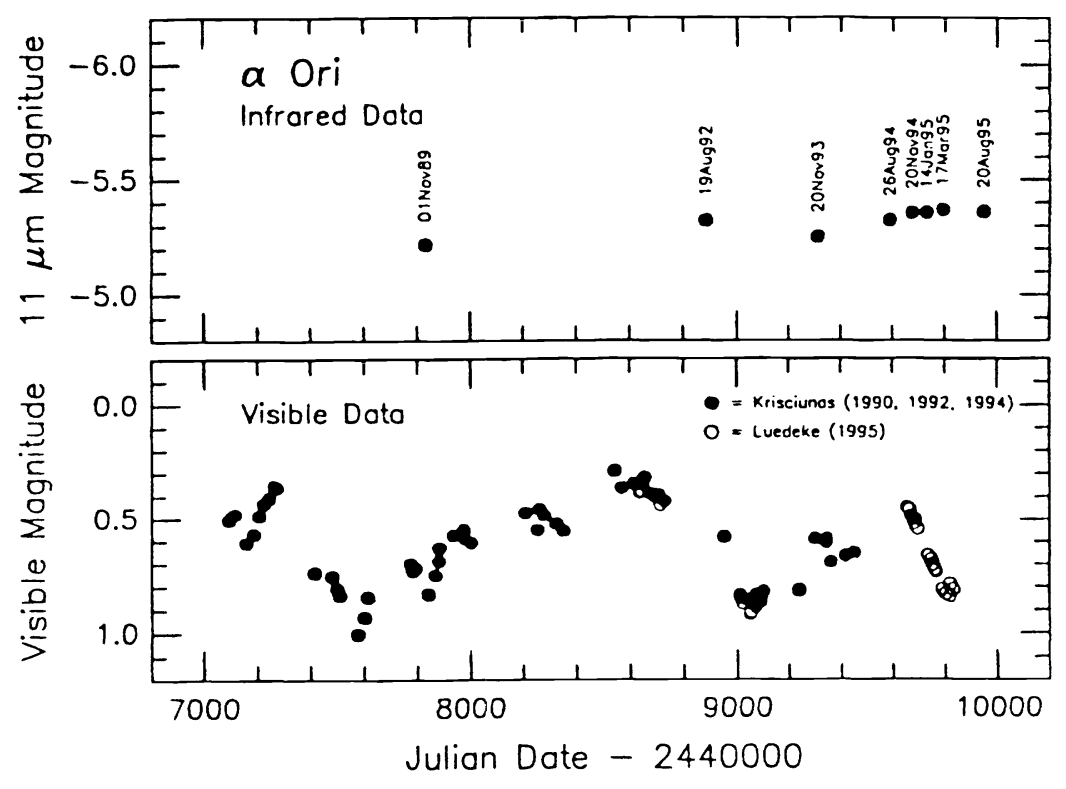

Fig. 19. Variations in the visible and $11 \mu \mathrm{m}$ magnitudes of $\alpha$ Orionis over a period of years. Before 1994, the IR luminosity varies synchronously with that of visible light, but by much smaller amounts. Beginning in 1994, the IR luminosity becomes slightly greater while that of visible light decreases markedly, indicating an emission of gas and dust. ${ }^{2}$

Another example of episodic emission of dust is demonstrated by the visibility curve of NML Cygni, shown in Fig. 20. ${ }^{10}$ Here the lower resolution points come from Dyck and Benson, ${ }^{4}$ and from Fix and Cobb. ${ }^{5}$ The higher resolution points were obtained more recently with the ISI system. What is notable is that the curve does not continue downwards smoothly but has a substantial bump. This indicates an irregularity in the distribution of dust. Fig. 21 shows a theoretical fit for this visibility curve. It plots the $11 \mu \mathrm{m}$ flux as a function of distance from the star, and shows peaks at about 90 mas and 340 mas. The solid curve represents a maximum entropy fit to the visibility data points. The other curves are somewhat idealized models, for example of two shells which are Gaussian in form. Measurement of the velocity of molecular gas around the star combined with its presumed distance allows one to calculate the separation in time between emission of the two shells, which is about 80 years.

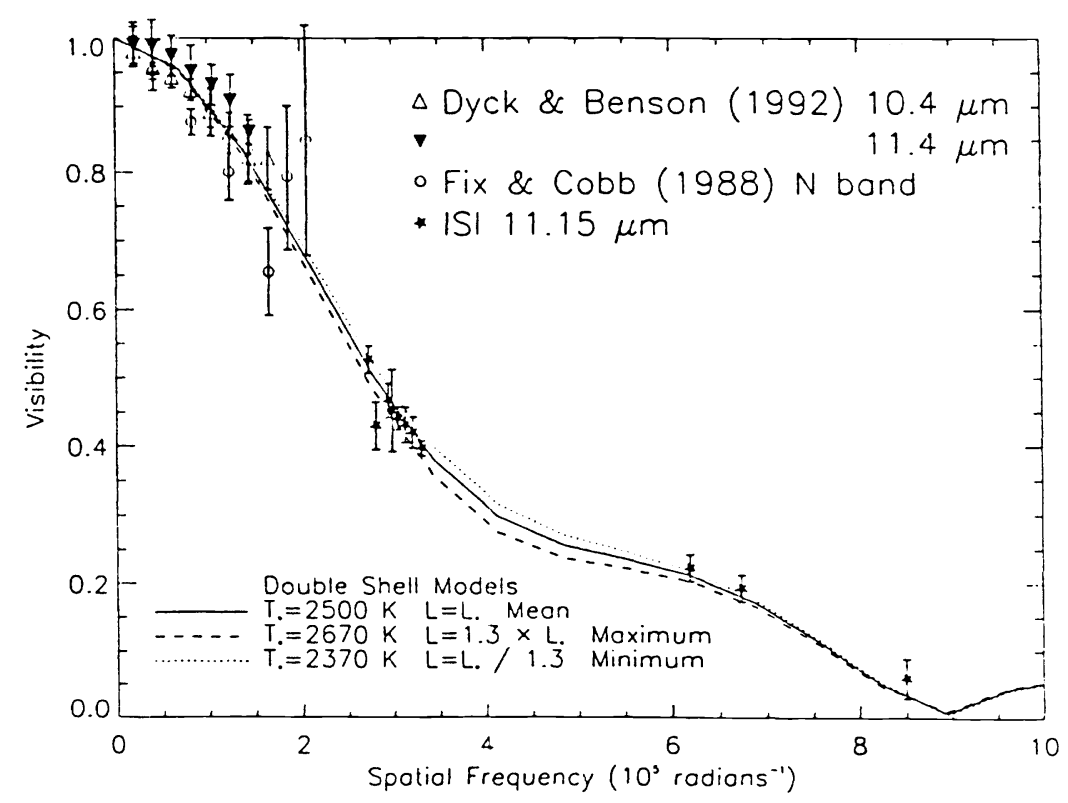

Fig. 20. The $11 \mu \mathrm{m}$ visibility curve of NML Cygni, indicating discrete shells of dust. 


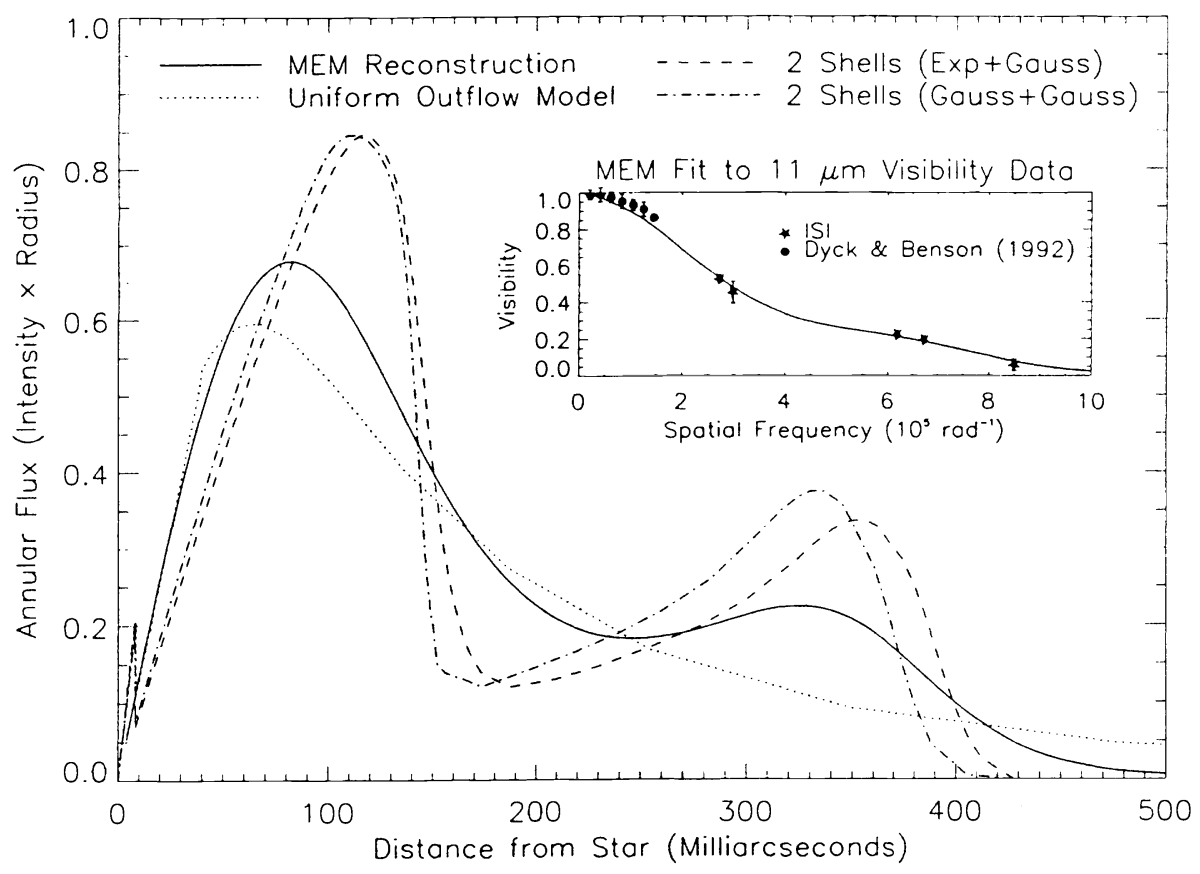

Fig. 21. Radial intensity distributions which give theoretical fits to the $11 \mu \mathrm{m}$ visibility curve of NML Cygni. These show two more or less discrete dust shells at distances of about 90 and 340 mas from the star. The solid curve is a maximum entropy fit to the visibility measurements. The other curves, which provide approximate fits, use somewhat different dust shell models.

Another case of episodic emission is IK Tau, for which the visibility curve is shown in Fig. $22 .{ }^{6}$ This visibility curve not only has some bumps, it changed noticeably over one year's time, between the fall of 1992 and the Fall of 1993. A maximum entropy fit for the intensity distribution as a function of distance from the star is shown in Fig. 23. It can be seen that the outer two peaks in intensity, corresponding to shells surrounding the stars, increased in distance between 1992 and 1993. If velocity measured for $\mathrm{OH}$ masers and $\mathrm{CO}$ gas around the star give the correct velocity of expansion of these shells, then the amount of expansion in one year's time allows an evaluation of the distance to the star, which is 265 parsec. $^{6}$ This corresponds rather well to previous estimates of distance, which were 270 and 220 parsec. With this distance and velocity, we can also determine the time between emission of the three successive shells indicated in this figure. That time is about 12 years. In the case of IK Tau, masking interferometry in the near infrared on the Keck telescope shows that the dust shell is rather spherical, at least close to the star. Hence in this case a spherical model for the outer shells is probably justified.

The visibility curves of o Ceti, shown in Fig. 24, are quite complex. ${ }^{8}$ The visibility changes not only with phase, but it does not repeat with successive cycles. This can be seen by comparing the top and bottom figures for visibility of o Ceti, both of which are near a minimum of luminosity but are taken at different cycles. The central diagram in this figure gives the visibility at maximum luminosity, which is also different from the other two. A detailed analysis and discussion of the case of o Ceti has been given by Lopez. ${ }^{8}$ This is too complex to discuss briefly here. However, it is found that there are nonsphericities in the distribution, and not only creation of new dust during the cool part of the star cycle, but probably evaporation of dust already formed during some of its higher luminosity phases.

It is clear that for a reasonably complete picture of the dust emission and distribution around many stars, a twodimensional interferometric picture must be made on a relatively short timescale, that is, a timescale of less than a few months, substantially shorter than the stellar period for variable stars. Fig. 25 shows a series of visibility measurements made on R Aqr. Since these include measurements made at a number of different times, the points do not fit on any simple single curve. Furthermore, they differ with each other even when the phases are the same. Thus, measurements taken in this way over a long period of time can only show the broad characteristics of the dust distribution, and not many of its intricate details. This makes it important for such measurements to have available multiple telescopes and baselines in the future. 


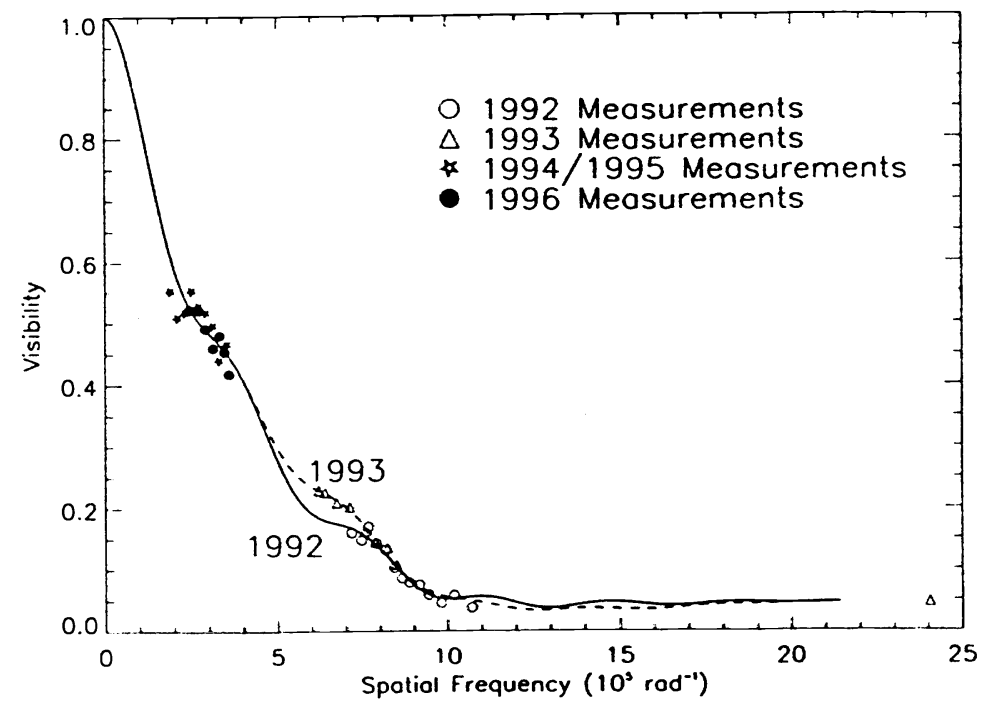

Fig. 22. Visibility curves of IK Tau for $11 \mu \mathrm{m}$ radiation. Two maximum entropy fits to the data of 1992 and of 1993 respectively are shown, since a distinct change appeared between these two years for spatial frequencies in the $5-7 \times 10^{5} \mathrm{rad}^{-1} \mathrm{range}_{\text {. }}$

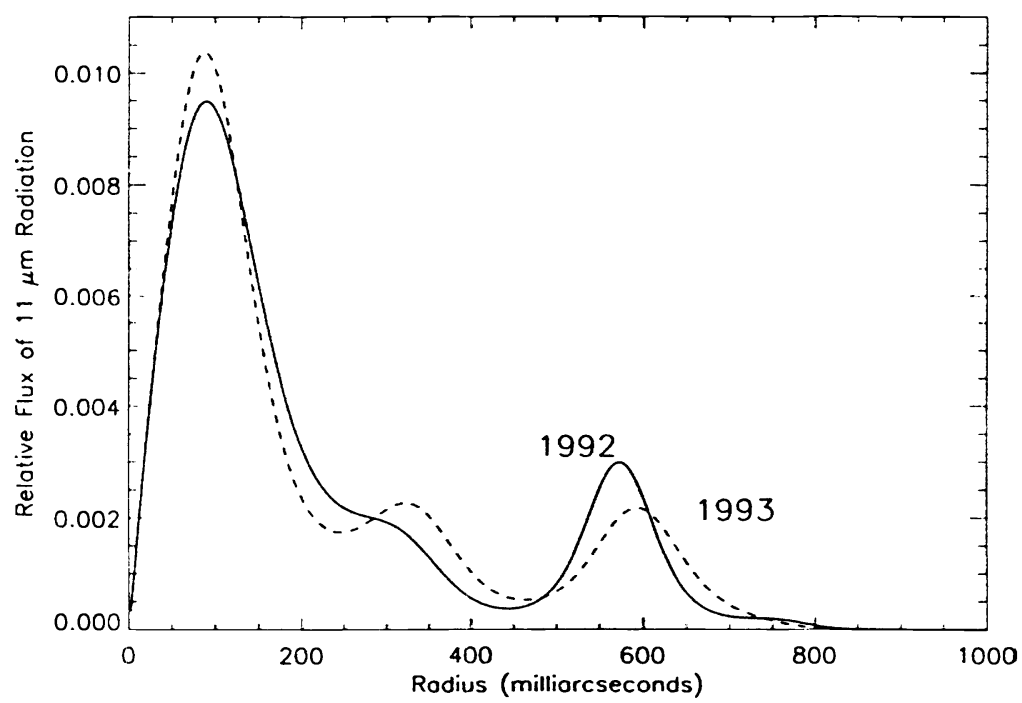

Fig. 23. The radial intensity distribution of $11 \mu \mathrm{m}$ radiation from dust around IK Tau. The two curves are maximum entropy fits of the two sets of points for 1992 and 1993. They show an outward motion of the outer two dust shells during one year's time. This allows a determination of the distance to the star $(265 \mathrm{pc})$ and the elapsed time between shells ( 12 years). 


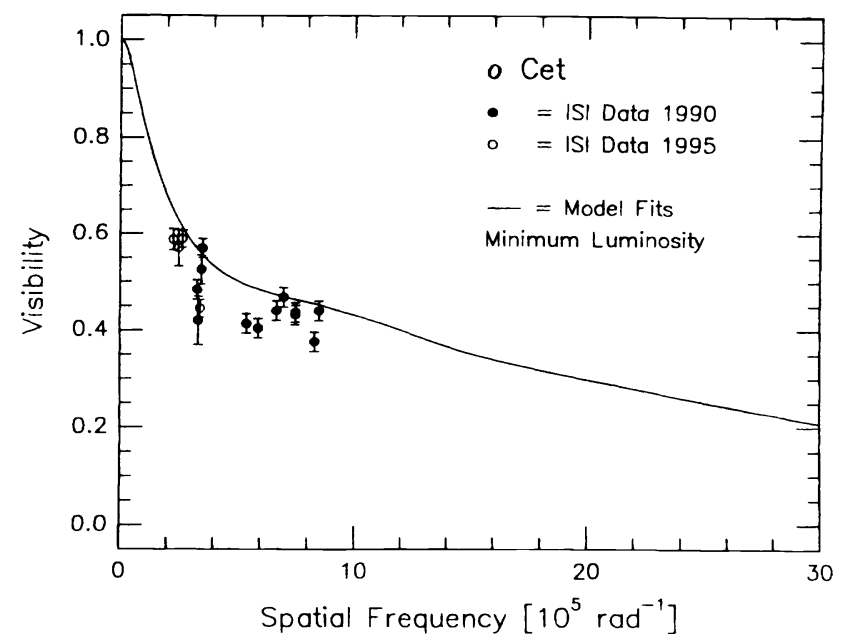

FIG. $7 a$

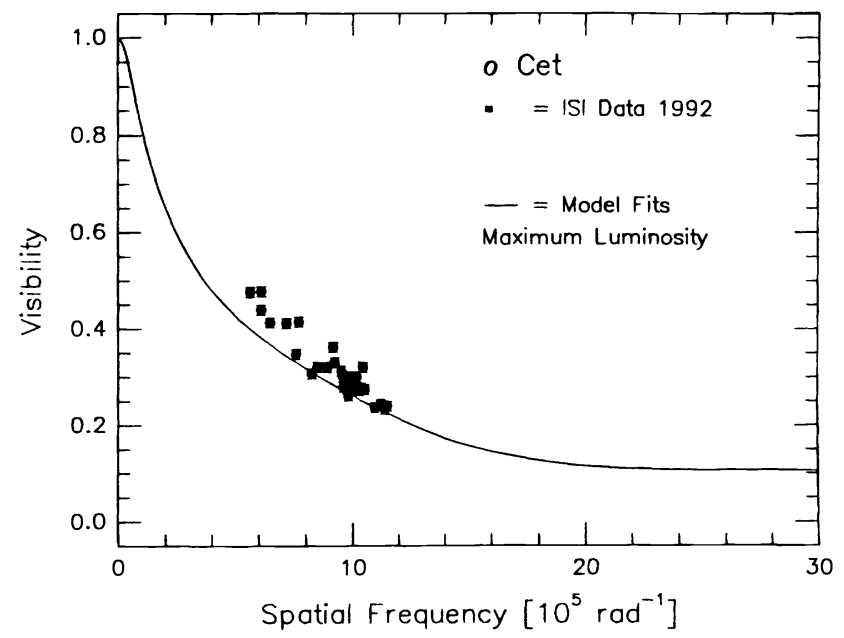

FıG. $7 b$

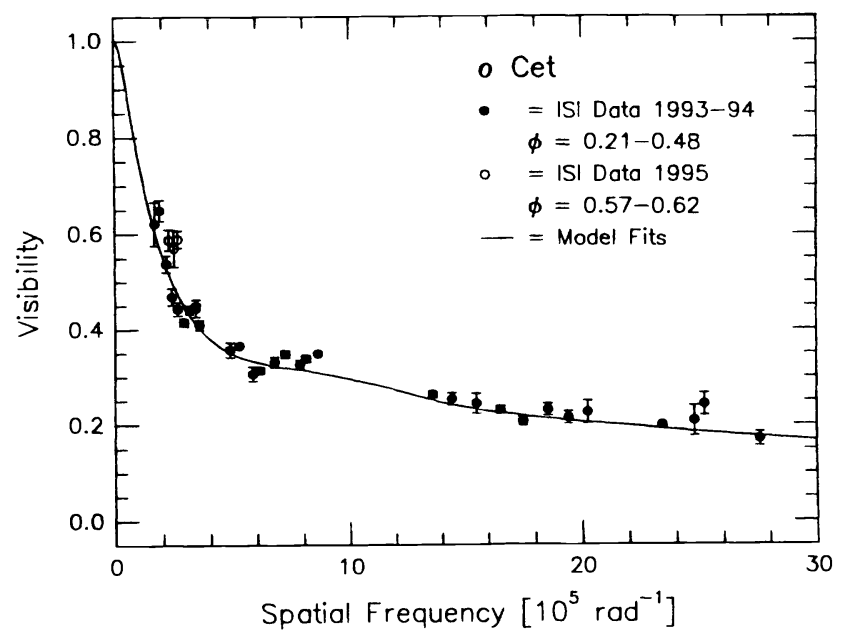

Fig. 24. Three $11 \mu \mathrm{m}$ visibility curves for o Ceti, showing both a variation with phase of this variable star and a variation in dust distributions at the same phase but during different cycles. 


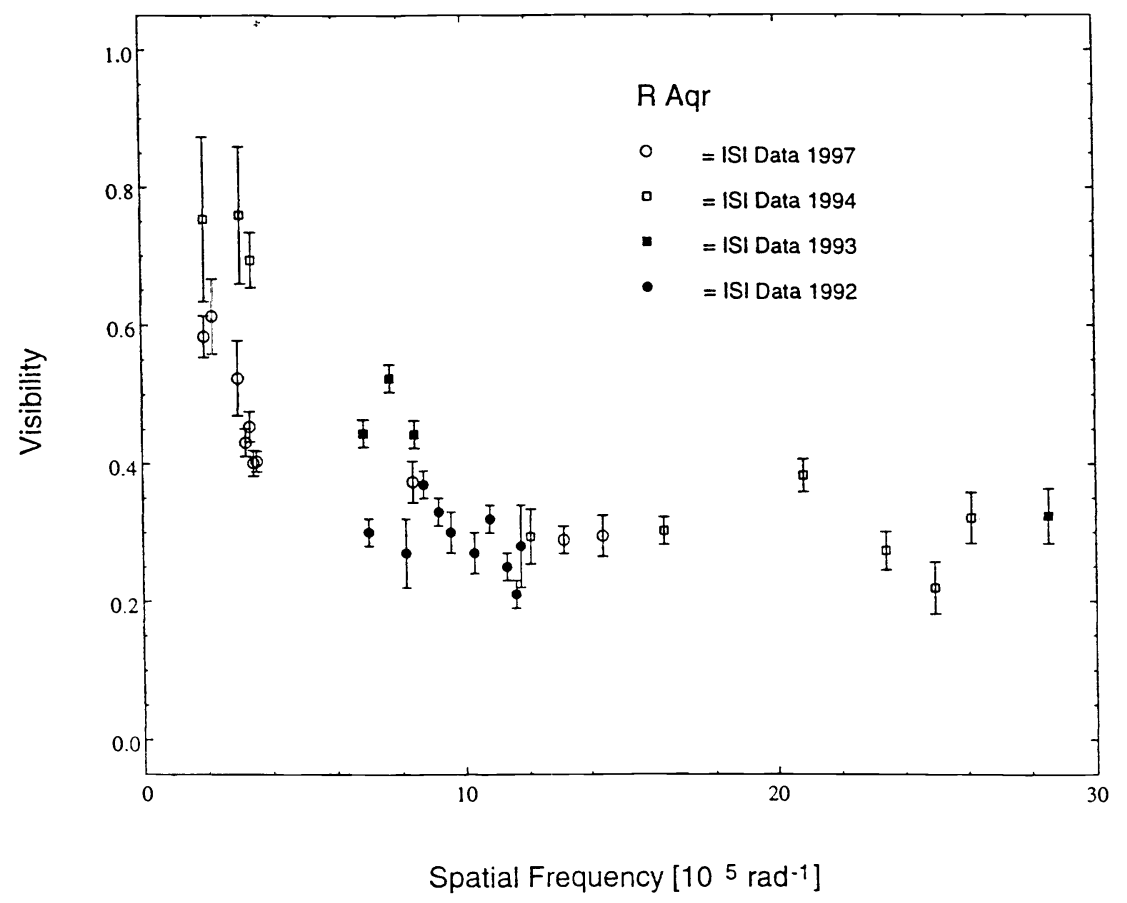

Fig. 25. A series of visibility measurements at $11 \mu \mathrm{m}$ of $\mathrm{R}$ Aquarii at different phases and different cycles. This shows variations in visibility which are often encountered, even at similar phases. It illustrates that, while such measurements can provide a gross picture of dust distribution around a star, an accurate measure requires a number of more or less simultaneous measurements in the UV plane. Such rapid and complete measurements are required to provide a detailed map of dust distribution and behavior for a number of stars which are variable and do not repeat a fixed pattern as they vary.

\section{STELLAR DIAMETERS}

The diameters of stars have been measured for some time by interferometry in the visible region; the first was of course the measurement of $\alpha$ Orionis by Michelson and Pease in 1921. ${ }^{9}$ The diameter of this star has been measured many times since then. A plot of various values obtained is shown in Fig. 26. Here, the probable errors given are clearly substantially less than variations in the results. White ${ }^{14}$ has made this plot in order to examine whether the size of $\alpha$ Orionis varied with its luminosity phase, and claimed that there was such evidence. However, it seems clear now that these measurements have probably been misleading due to nonuniformities and changes in intensity distribution over the star.

Visibility curves for stars at visible wavelengths have characteristically been fitted to a uniformly intense disk, and stellar diameters thereby assigned. It has been recognized for some time that these disks are not uniform but are darkened on the outer edges by what is called "limb darkening," and that furthermore this limb darkening typically reduces the apparent size of the star in the visible region by about $10 \% .{ }^{11}$ This $10 \%$ estimate comes from theoretical calculations in the past, rather than actual measurements. There is now experimental evidence, however, for such limb darkening. ${ }^{2,7}$ Recently, it has been recognized that older stars in particular can vary in intensity over the disk by having hot spots, somewhat similar to solar sunspots but very much larger in magnitude. Certain of these hot spots can produce a substantial fraction of the total intensity, and thereby decrease the apparent size of the star if a simple fit to a limited number of points on the visibility curve is made, and a uniform disk is assumed. There have also been claims that stars vary in shape, with some substantially elongated as well as changing in size. We believe these results may be questionable and need to be checked with infrared measurements. The infrared intensity is much less sensitive to changes in temperature on the surface of the star, as pointed out above, and hence generally will represent a rather uniform disk. In addition, the limb darkening is much reduced in the infrared region. At 10 
$\mu \mathrm{m}$, calculations indicate that it represents only about a $1 \%$ decrease in the apparent size. In addition, if there is dust around a star or certain types of gas, then obscuration or scattering can substantially affect the apparent size of the star in the visible region, while not affecting it very much at the longer IR wavelengths. One can already deduce, from Fig. 19, that, as the visible luminosity changes, the change is primarily due to a change in temperature rather than a change in size of $\alpha$ Orionis, since the visible changes in luminosity are very much greater than those in the infrared. Hence, it seems likely that the large changes in the size of $\alpha$ Orionis indicated by Fig. 26 are quite misleading. Stellar size measurements at IR wavelengths can also be misleading if a star's atmosphere contains molecular gas which absorbs infrared wavelengths, and such effects need exploration.

We have measured the size of $\alpha$ Orionis at $11 \mu \mathrm{m}$ from the visibility curve shown in Fig. 18. The size of $\alpha$ Sco has also been measured. ${ }^{2}$ These sizes are shown in Table 1 . The table also shows a measurement of the visible size of $\alpha$ Ori. This is a measurement which used phase closure and high resolution to take into account a spot on the star. ${ }^{13}$ We believe this is the most reliable measurement of the size of $\alpha$ Orionis in the visible region. Our own measurements at $11 \mu \mathrm{m}$ give a result of $56 \pm 1$ mas, which is close to the theoretically estimated $9 \%$ increase in size to be expected from the visible to $11 \mu \mathrm{m}$ wavelengths. Similar results were found for $\alpha$ Sco, as shown in Table 1 . Although these results appear to fit limb darkening expectations, further measurements of stellar sizes at a variety of wavelengths is needed in order to examine details of limb darkening and of the transparency of stellar atmospheres.

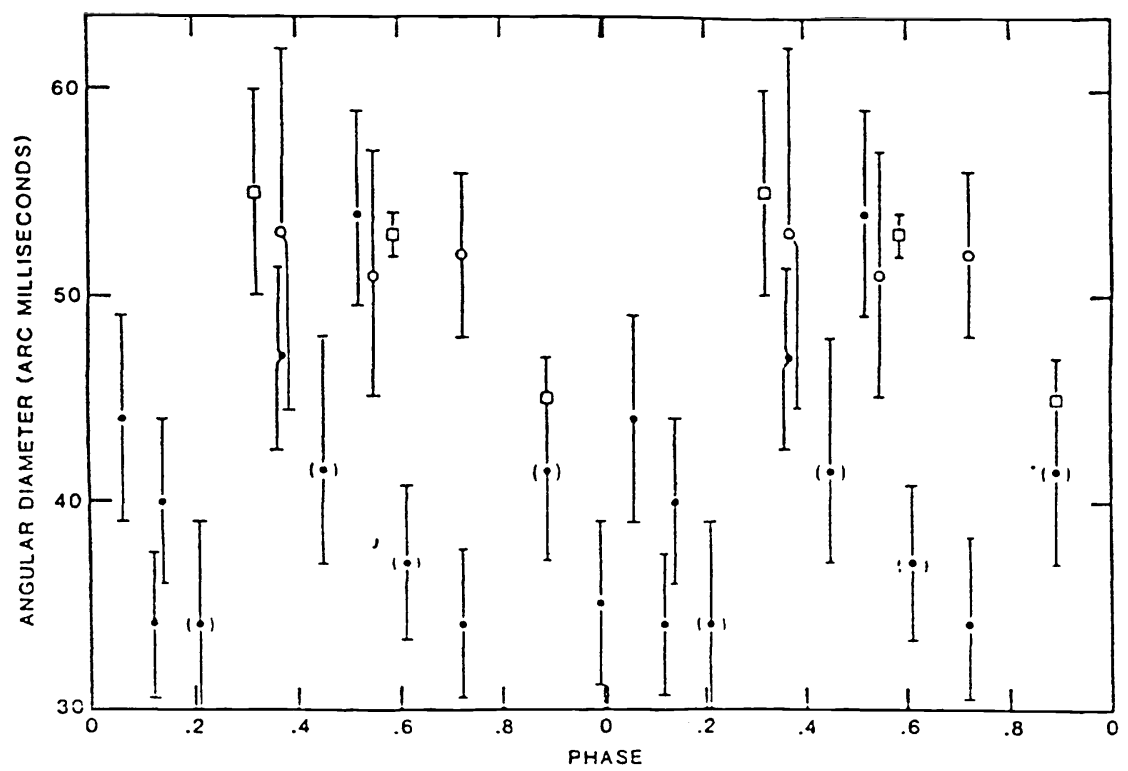

Fig. 26. Various measurements of the diameter of $\alpha$ Orionis by interferometry at visible wavelengths. These have been arranged by White ${ }^{14}$ in accordance with the apparent phase of $\alpha$ Ori's somewhat irregular variations in luminosity. It is clear that the apparent size variation is substantially larger than the stated probable errors of measurement. This now appears to be primarily due to non-uniformity and change of intensity distributions over the stellar surface. 
Table 1

Measured diameters of $\alpha$ Ori and $\alpha$ Sco

\begin{tabular}{|c|c|c|c|}
\hline Objects & $\begin{array}{l}\mathrm{D}_{\text {vis }} \\
\text { Diameter at visible } \\
\text { wavelengths without } \\
\text { limb darkening correction }\end{array}$ & $\begin{array}{l}\mathrm{D}_{11 \mu \mathrm{m}} \\
\text { Diameter at } 11 \mu \mathrm{m} \\
\text { wavelength without limb } \\
\text { darkening correction }\end{array}$ & $\begin{array}{l}\text { D } \\
\text { Actual diameter } \\
\text { estimated from } 11 \mu \mathrm{m} \\
\text { value with } 1 \% \text { correction } \\
\text { for limb darkening }\end{array}$ \\
\hline$\alpha$ Ori & $52.7 \pm 1.3 \mathrm{mas}$ & $56.0 \pm 1 \mathrm{mas}$ & 56.6 mas \\
\hline$\alpha$ Sco & $40.0 \pm 0.6 \mathrm{mas}$ & $44.0 \pm 2$ mas & 44.4 mas \\
\hline
\end{tabular}

With the infrared size measurements comes an additional interpretation of stellar temperatures. The temperature is of course not constant in all parts of the star, and hence can depend on characteristics of stellar atmospheres and how such temperatures are defined. Table 2 shows a collection of temperatures, some using the diameters measured at $11 \mu \mathrm{m}$. We believe these represent useful and representative values of the temperature.

Table 2

Temperatures of $\alpha$ Ori and $\alpha$ Sco

\begin{tabular}{llll} 
& $\begin{array}{l}\mathrm{T}_{\text {vis }} \\
\text { Effective temperature from } \\
\text { luminosity and } \\
\text { assuming } \mathrm{D}_{\text {vis }}\end{array}$ & $\begin{array}{l}\mathrm{T}_{11 \mu \mathrm{m}} \\
\text { Effective temperature } \\
\text { from luminosity and } \\
\text { assuming } \mathrm{D}_{11 \mu \mathrm{m}}\end{array}$ & $\begin{array}{l}\mathrm{T} \\
\text { Temperature calculated } \\
\text { from } 11 \mu \mathrm{m} \text { flux and } \\
\mathrm{D}_{11 \mu \mathrm{m}}\end{array}$ \\
\hline $\begin{array}{l}\text { Obji } \\
\alpha \text { Sco }\end{array}$ & 3290 & 3190 & 2850 \\
& 3670 & 3500 & 2675
\end{tabular}




\section{CONCLUSION}

A broad examination of the results of ISI observations of a number of stars leads to the following conclusions:

1) Dust is formed around the stars at temperatures between 1,000 and $1,500 \mathrm{~K}$, approximately as expected theoretically.

2) About half of the stars observed emit dust episodically rather than continually or on each luminosity cycle.

3) The time between episodic emissions varies from about 10 years to about 100 years. A few stars around which several shells can be seen appear to have shells approximately equally separated, indicating perhaps a periodicity. However, this conclusion is not a secure one. What we do know is that these times are long, and vary substantially between the stars. There seems to be no current theory which can produce timescales of this general magnitude.

4) Cycles of variable stars clearly do not repeat very precisely. This has been known previously for some stars because of a lack of repetition of the visible magnitude as a function of the phase of a cycle. However, we can now see that this is also associated with variations in the amount of dust emitted or surrounding the star at any one time.

5) Clearly many stars emit dust in patterns which are not spherically symmetric. It is not yet clear whether this nonsymmetry can be directly associated with certain types of stars only, and further study will be needed to examine this question.

6) It appears important and fruitful to measure the diameter of stars in the infrared, perhaps in the near infrared as well as the mid-infrared. This should produce a more reliable and accurate picture of whether the stars are changing size and shape as well as give more representative diameters and additional insight into their atmospheres and temperatures.

7) It is important to make rapid interferometric measurements and over a substantial part of the UV plane in order to get reasonably complete pictures of the distribution and behavior of dust. The distributions are not spherical, they can change rather rapidly with time, and are not completely periodic. This emphasizes the importance of our building a third telescope so that we can obtain somewhat more rapid and more complete visibility measurements.

As we look to the future, we expect soon to do interferometry on spectral lines. Fig. 27 shows absorption lines of silane in IRC +10216 and of ammonia in NML Cygni. Interferometry on their line frequencies can help establish where these molecules are formed. This may be particularly interesting because circumstellar material represents one of the few cases where the circumstances under which molecules are formed can be known. Material emerges from the star in more or less atomic form and becomes molecular as it cools off and material condenses into dust. Thus the timing, mechanisms, and nature of the formation can probably be examined in some detail. We have built filters for our heterodyne interferometry system so that visibility measurements can be made in narrow bands both on and off a line. The filters are somewhat narrower in frequency range than are the lines, so that various parts of these lines can be examined. This should allow determination of the distribution of molecular material and the distribution of excitation and velocity. We hope and expect that in the future this too will provide interesting and revealing information. 

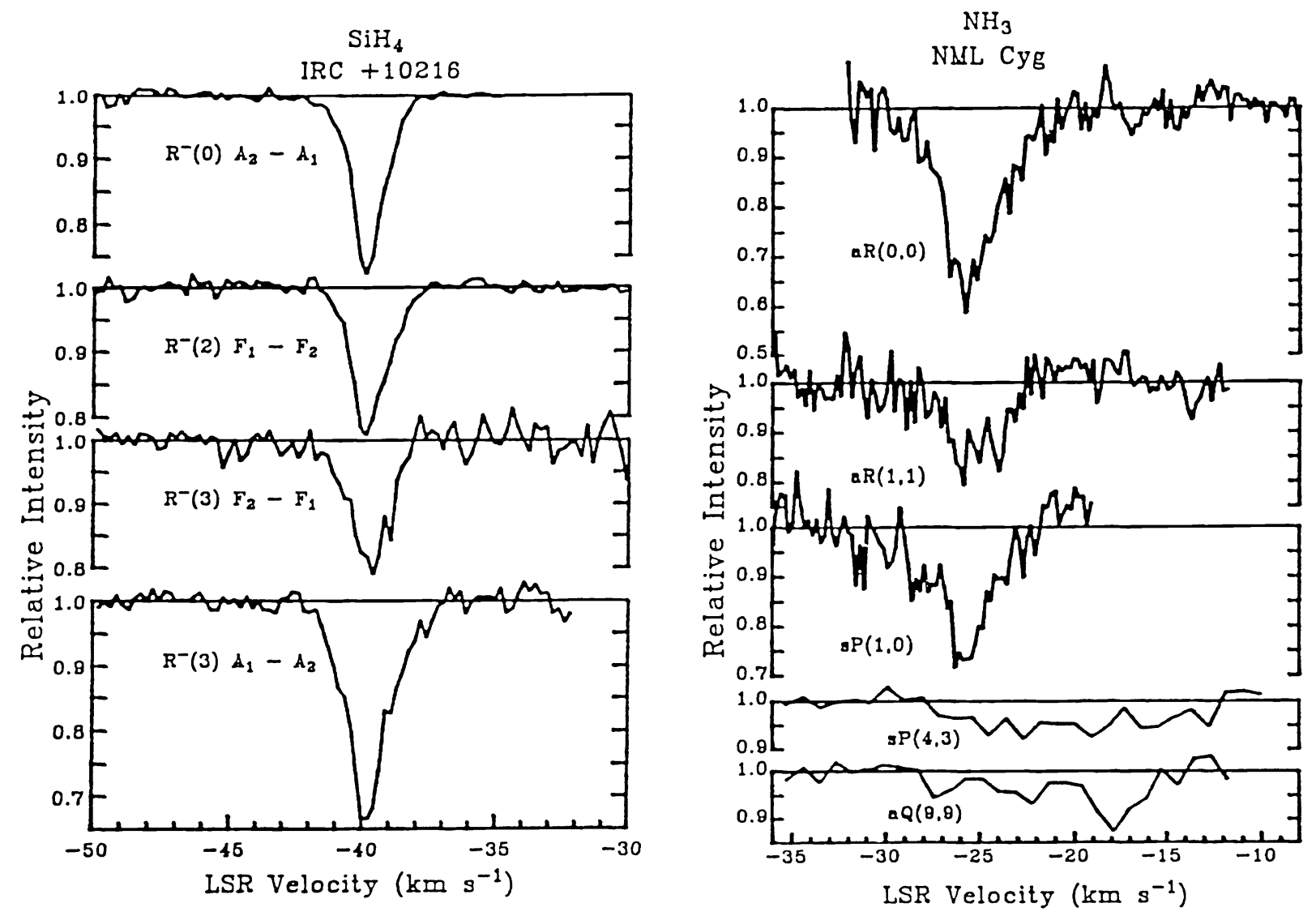

Fig. 27. Spectral lines of $\mathrm{SiH}_{4}$ and $\mathrm{NH}_{4}$ due to formation of such molecules in material emitted by stars such as IRC +10216 and NML Cyg. The fractional intensity is plotted here as a function of Doppler shift due to a velocity with respect to the local standard of rest (LSR). It is expected that high angular resolution by interferometry in the mid-IR region will help determine the places of appearance of various excitation states of these molecules, and allow some quantitative tests of theories of formation. 


\section{ACKNOWLEDGMENTS}

This work was supported by grants from the Office of Naval Research (grants N00014-96-0737 and N00014-97-10743), and from the National Science Foundation (grants AST 9321289, AST 9321384, and AST 9500525). M.J. was supported under the auspices of the U.S. Department of Energy, contract W-7405-ENG-48. D.W. was supported under the auspices of the U.S. Department of the Navy.

\section{REFERENCES}

1. M. Bester, W.C. Danchi, and C.H. Townes, "Long baseline interferometer for the mid-infrared," Amplitude and Intensity Spatial Interferometry, J.B. Breckinridge (ed.), Proc. SPIE 1237, pp. 40-48, 1990.

2. M. Bester, W.C. Danchi, D. Hale, C.H. Townes, C.G. Degiacomi, D. Mékarnia, and T.R. Geballe, "Measurement at 11 micron wavelengths of the diameters of $\alpha$ Orionis and $\alpha$ Scorpii; and changes in effective temperature of $\alpha$ Orionis and very recent dust emission," ApJ 463, pp. 336-343, 1996.

3. W.C. Danchi, M. Bester, C.G. Degiacomi, and L.J. Greenhill, and C.H. Townes, "Characteristics of dust shells around 13 late-type stars," $A J 107$, pp. 1469-1513, 1994.

4. M.M. Dyck and J.A. Benson, "Variations in the 8-13 $\mu \mathrm{m}$ visibility functions of shells around oxygen rich stars," $A J$ 104, pp. 377-385, 1992.

5. J.D. Fix and M.L. Cobb, "The structure of circumstellar shells," ApJ 329, pp. 290-298, 1988.

6. David D.S. Hale, M. Bester, W.C.Danchi, S. Hoss, E. Lipman, J.D. Monnier, P.G. Tuthill, C.H. Townes, M. Johnson, B. Lopez, and T.R. Geballe, "Multiple dust shells and motions around IK Tauri as seen by infrared interferometry," $A p J$ 490, pp. 407-411, 1997.

7. A.R. Hajian, J.T. Armstrong, C.A. Hummel, J.A. Benson, D. Mozurkevich, T.A. Pauls, D.J. Hutter, N.M. Elias II, K.J. Johnston, L.J. Richard, and N.M. White, "Direct confirmation of stellar limb darkening with the Navy prototype optical interferometer," ApJ 496, pp. 484-489, 1998.

8. B. Lopez, et al, "Nonspherical structures and temporal variations in the dust shell of o Ceti observed with a long baseline interferometer at 11 microns," ApJ 488, pp. 807-826, 1997.

9. A.A. Michelson and P.G. Pease, "Measurement of the diameter of $\alpha$ Orionis with the interferometer," $A p J 53$, pp. 249-259, 1921.

10. J.D. Monnier, M. Bester, W.C. Danchi, M.A. Johnson, E.A. Lipman, C.H. Townes, P.G. Tuthill, T.R. Geballe, D. Nishimoto, and P.W. Kervin, "Nonuniform dust outflow observed around the infrared object NML Cygni," ApJ 481, pp. 420-432, 1997.

11. S.T. Ridgway, D.C. Wells, and R.R. Joyce, "Angular diameters for 11 late-type stars by the lunar occultation technique," AJ 82, pp. 414-430, 1977.

12. R.N. Treuhaft, S.T. Lowe, M. Bester, and W.C. Danchi, and C.H. Townes, "Vertical scales of turbulence at the Mount Wilson Observatory," ApJ 453, pp. 522-531, 1995.

13. P.G. Tuthill, "Imaging stars through the atmosphere," Ph.D. thesis, Cambridge Univ., 1994.

14. N.M. White, "The occultation of 119 Tauri and the effective temperatures of three M supergiants," ApJ 242, $646,1980$. 